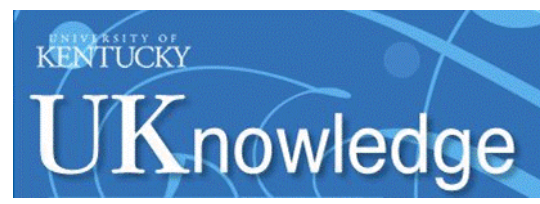

University of Kentucky

UKnowledge

$10-3-2011$

\title{
Critical Differences and Clues in Eta Car's 2009 Event
}

\author{
Andrea Mehner \\ University of Minnesota \\ Kris Davidson \\ University of Minnesota \\ John C. Martin \\ University of Illinois \\ Roberta M. Humphreys \\ University of Minnesota \\ Kazunori Ishibashi \\ Nagoya University, Japan
}

See next page for additional authors

Follow this and additional works at: https://uknowledge.uky.edu/physastron_facpub

Part of the Astrophysics and Astronomy Commons, and the Physics Commons

Right click to open a feedback form in a new tab to let us know how this document benefits you.

\section{Repository Citation}

Mehner, Andrea; Davidson, Kris; Martin, John C.; Humphreys, Roberta M.; Ishibashi, Kazunori; and Ferland, Gary J., "Critical Differences and Clues in Eta Car's 2009 Event" (2011). Physics and Astronomy Faculty Publications. 70.

https://uknowledge.uky.edu/physastron_facpub/70

This Article is brought to you for free and open access by the Physics and Astronomy at UKnowledge. It has been accepted for inclusion in Physics and Astronomy Faculty Publications by an authorized administrator of UKnowledge. For more information, please contact UKnowledge@lsv.uky.edu. 


\section{Critical Differences and Clues in Eta Car's 2009 Event}

Digital Object Identifier (DOI)

https://doi.org/10.1088/0004-637X/740/2/80

\section{Notes/Citation Information}

Published in The Astrophysical Journal, v. 740, no. 2, 80, p. 1-19.

(c) 2011. The American Astronomical Society. All rights reserved. Printed in the U.S.A.

The copyright holder has granted permission for posting the article here.

Authors

Andrea Mehner, Kris Davidson, John C. Martin, Roberta M. Humphreys, Kazunori Ishibashi, and Gary J. Ferland 


\title{
CRITICAL DIFFERENCES AND CLUES IN ETA CAR'S 2009 EVENT*,†
}

\author{
Andrea Mehner $^{1}$, Kris Davidson $^{1}$, John C. Martin ${ }^{2}$, Roberta M. Humphreys $^{1}$, \\ KAZUNORI ISHIBASHI ${ }^{3}$, AND GARY J. FERLAND ${ }^{4}$ \\ ${ }^{1}$ Department of Astronomy, University of Minnesota, Minneapolis, MN 55455, USA \\ 2 Astronomy-Physics Department, University of Illinois Springfield, Springfield, IL 62703, USA \\ ${ }^{3}$ Department of Physics, Nagoya University, Nagoya 464-8602, Japan \\ ${ }^{4}$ Department of Physics \& Astronomy, University of Kentucky, Lexington, KY 40506, USA \\ Received 2011 June 20; accepted 2011 July 19; published 2011 October 3
}

\begin{abstract}
We monitored Eta Carinae with the Hubble Space Telescope WFPC2 and Gemini GMOS throughout the 2009 spectroscopic event, which was expected to differ from its predecessor in 2003. Here we report major observed differences between events and their implications. Some of these results were quite unexpected. (1) The UV brightness minimum was much deeper in 2009. This suggests that physical conditions in the early stages of an event depend on different parameters than the "normal" inter-event wind. Extra mass ejection from the primary star is one possible cause. (2) The expected He II $\lambda 4687$ brightness maximum was followed several weeks later by another. We explain why this fact and the timing of the $\lambda 4687$ maxima strongly support a "shock breakup" hypothesis for X-ray and $\lambda 4687$ behavior as proposed 5-10 years ago. (3) We observed a polar view of the star via light reflected by dust in the Homunculus nebula. Surprisingly, at that location, the variations of emission-line brightness and Doppler velocities closely resembled a direct view of the star, which should not have been true for any phenomena related to the orbit. This result casts very serious doubt on all the proposed velocity interpretations that depend on the secondary star's orbital motion. (4) Latitude-dependent variations of $\mathrm{H}$ I, He I, and Fe II features reveal aspects of wind behavior during the event. In addition, we discuss implications of the observations for several crucial unsolved problems.
\end{abstract}

Key words: circumstellar matter - stars: emission-line, Be - stars: individual (Eta Carinae) - stars: variables: general - stars: winds, outflows

Online-only material: color figure

\section{INTRODUCTION}

Eta Carinae is Exhibit A for episodic mass loss near the top of the H-R diagram, for the physics of giant eruptions ("supernova impostors") and subsequent recovery, the behavior of outflows above the Eddington limit, polar winds, several exotic nebular processes, etc. Most of these topics remain poorly understood, but there is no reason to think that $\eta$ Car structurally differs from other extremely massive stars. A likely companion object affects the phenomena, but does not by itself constitute an "explanation."

Beginning in the mid-1940s $\eta$ Car began to exhibit occasional spectroscopic changes that we now recognize as a 5.5 year spectroscopic/photometric cycle. Occasionally, its high-excitation $\mathrm{He}$ I, [Ne III], [Fe III] emission lines disappear for a few weeks or months (Gaviola 1953; Zanella et al. 1984) while other changes also occur, specifically in the X-ray (e.g., Corcoran et al. 1997; Ishibashi et al. 1999a, 1999b) and infrared flux (e.g., Whitelock et al. 1994; Feast et al. 2001). These spectroscopic events recur with a period close to 2023 days (Damineli 1996; Whitelock et al. 1994, 2004; Damineli et al. 1999, 2000, 2008b; Martin et al. 2006a; Fernández-Lajús et al. 2010). They have been attributed to (1) eclipses of a hot sec-

\footnotetext{
* Based on observations obtained at the Gemini Observatory, which is operated by the Association of Universities for Research in Astronomy, Inc., under a cooperative agreement with the NSF on behalf of the Gemini partnership.

$\dagger$ Based on observations made with the NASA/ESA Hubble Space Telescope. STScI is operated by the Association of Universities for Research in Astronomy, Inc., under the NASA contract NAS 5-26555.
}

ondary star by the primary wind (Damineli et al. 1997; Ishibashi et al. 1999b; Stevens \& Pittard 1999; Pittard \& Corcoran 2002), (2) disturbances in the primary wind triggered by a companion star near periastron (Davidson 1997, 1999; Smith et al. 2003; Martin et al. 2006a), (3) a thermal/rotational recovery cycle (Zanella et al. 1984; Davidson et al. 2000; Smith et al. 2003; Davidson 2005), or (4) a breakup/collapse of the wind-wind collision structure due to known shock instabilities (Davidson 2002; Soker 2003; Martin et al. 2006a; Soker \& Behar 2006). These ideas are not mutually exclusive. Either (2) or (3) would be significant for massive-star physics in general because they may require an undiagnosed instability near the Eddington limit.

Observations of the 2003.5 event appeared to favor possibility (2) and especially (4), but did not rule out number (3), and the likely geometry suggests that an eclipse probably occurred with lesser consequences. Photometric behavior, a chaotic X-ray behavior, and an unpredicted He II $\lambda 4687$ outburst (Steiner \& Damineli 2004; Martin et al. 2006a) were especially significant.

Meanwhile the longer-term behavior changed dramatically. The central star brightened rapidly after 1998 (Davidson et al. 1999a; Martin \& Koppelman 2004; Martin et al. 2006b, 2010), and major spectral features differed between the 1998.0 and 2003.5 events (Davidson et al. 2005). Destruction and/or lessened formation of dust played a role, but the root cause must have involved a secular change in the UV flux or the wind density or both. Thus, from the viewpoint of 2007-2008, observations of the expected 2009.0 event merited a high priority for comparisons with 2003.5 and 1998.0. Unfortunately, the Space Telescope Imaging Spectrograph on the Hubble Space Telescope (STIS HST) had failed in 2004, and thus was not 
available to separate the star from ejecta (see below). Later, after the event was observed as discussed in this paper, STIS became operational again and proved that emission lines from $\eta$ Car's wind had greatly weakened since 2004; the mass-loss rate had probably decreased by a factor of the order of two or three in a time span of several years (Mehner et al. 2010b; see also Kashi \& Soker 2009a and Corcoran et al. 2010). Therefore, the 2009.0 spectroscopic event occurred in physical circumstances appreciably different from its predecessors. This should be helpful for deducing the nature of the event, on the same principle as varying parameters in an experiment.

Ground-based spectra of $\eta$ Car represent an unresolved mixture of the central star plus bright ejecta located at $r \sim$ $0.2-2$ ". Fortunately, though, the central star has brightened more than the nearby ejecta in the past decade, so ground-based observations of it have become relatively less contaminated than at earlier times. ${ }^{5}$ Thus, in 2007 we began to observe the star and several offset positions in the Homunculus nebula with the Gemini-South Multi-Object Spectrograph (GMOS) on the Gemini-South telescope. Apart from the question of secular changes, we planned to measure some aspects of the spectroscopic event better than had been done in 2003 and earlier. A primary goal was to monitor the behavior of the peculiar He II $\lambda 4687$ line with more frequent observations during the 2009 event. We also observed the spectrum reflected by dust in the southeast (SE) Homunculus lobe, a "polar" view of the star.

In this paper, we discuss the resulting spectra and the light curve of the central star. Some aspects of the 2009 event have been reported by other authors: Richardson et al. (2010) described the behavior of $\mathrm{H} \alpha$, Kashi \& Soker (2009a) and Corcoran et al. (2010) commented on the X-rays, Groh et al. (2010) reported high-velocity material, Fernández-Lajús et al. (2010) discussed photometry, and Teodoro et al. (2011) have recently discussed the He II $\lambda 4687$ emission. Here our scope is broader. As we explain below, some of the differences compared to the 2003 event provide unexpected new evidence for specific phenomena, UV photometry of the central star indicates changed conditions, reflected spectra showing the polar view appear inconsistent with published models of the velocities, and we strongly disagree with Teodoro et al. concerning the past observational record and theoretical interpretations. Our main new conclusions are that (1) differences between the 2003 and 2009 events give valuable and specific clues to the phenomena, (2) a "shock breakup" scenario proposed a decade ago now seems almost inescapable, (3) the observed Doppler velocities are far less straightforward than most authors have assumed, and (4) realistic quantitative models - as opposed to qualitative scenarios or idealized simulations - are badly needed but will be very difficult to achieve. We also comment on many other observational and theoretical factors. Some important ideas that were discussed in connection with the 2003 event remain valid.

HST photometry and Gemini GMOS observations are described in the next section. The 2008-2010 light curve of the central star and the unusually deep minimum during the 2009 event are discussed in Section 3. In Section 4, we discuss the peculiar He II emission during the event, its connection with the X-ray flux curve, and the "FOS4" polar view of He II emission compared with our direct line-of-sight view. The changing wind geometry during the 2009 event is discussed in Section 5. In

\footnotetext{
5 Here "central star" really means the opaque primary wind. The secondary star is also included, but its spectrum is too faint to be observable at accessible wavelengths.
}

the discussion section, we summarize the results, emphasize the new information given by the 2009 event, and raise some outstanding questions.

\section{OBSERVATIONS AND DATA REDUCTION}

\subsection{HST Photometry with WFPC2 and STIS}

We have monitored the brightness changes of the central star in several bandpasses with photometry from HST Advanced Camera for Surveys High Resolution Camera (ACS/HRC) and WFPC2 images and STIS spectra since 1998 (Martin \& Koppelman 2004; Martin et al. 2006b, 2010). During the 2009 event we monitored the brightness of the central star with the HST WFPC2 camera using the F255W and F336W filters. F255W samples the NUV "Fe II forest" (Cassatella et al. 1979; Altamore et al. 1986; Viotti et al. 1989), which greatly increases in opacity during a spectroscopic event (Davidson et al. 1999b; Gull et al. 2000). F336W includes the Balmer continuum augmented by various emission lines. These filters have been calibrated for direct comparisons with the HST ACS/HRC F250W and F330W filters (Sirianni et al. 2005). Our own careful checks have led us to conclude that comparisons in these filters across instruments are valid for $\eta$ Car. The images were reduced using the standard STScI data reduction pipeline. Calibrated fluxes were measured in a $00^{\prime \prime} 3$ diameter weighted aperture following procedures described in our previous papers which, combined with the spatial resolution of the HST, minimize the influence from nearby bright ejecta. ACS-equivalent photometry was also synthesized from HST STIS data before mid-2004 and after mid-2009; spectra were extracted with a weighted parabolic cross-dispersion profile similar to the virtual aperture used to measure ACS/HRC images, convolved with the filter functions, and integrated (Martin et al. 2006b). The WFPC2 photometry from 2008.7 to 2009.3 and STIS synthetic photometry from 2009.6 to 2010.6 is listed in Table 1. Earlier data from ACS/HRC and WFPC2 images and STIS spectra can be found in the papers cited above.

\subsection{Gemini GMOS Observations}

To cover the 2009.0 event, we obtained ground-based slit spectroscopy of $\eta$ Car with the GMOS from 2007 June to 2010 January. In most cases, we used the B1200 line grating at three tilt angles to cover the spectrum from 3700 to $7500 \AA$. The $00^{\prime \prime} 5$ wide slit, oriented with a position angle of $160^{\circ}$, was placed at four different positions: on the star, two offset positions \pm 0 '.75 relative to the star, and at a position known as "FOS4," 4". 5 from the star in the SE lobe of the Homunculus. (Operationally, this was done by offsetting the slit $2^{\prime \prime}$ parallel to itself.) The star's polar spectrum is reflected by dust at FOS4. ${ }^{6}$

Since most of this paper is concerned with the He II $\lambda 4687$ emission and features at nearby wavelengths, we concentrate on the blue spectra of the star and FOS4. Table 2 is a journal of those observations. True slit positions vary slightly from the four locations described above, due to a combination of blind offset errors and differential atmospheric refraction, which were comparable. No corrector was available, and our observing goals

\footnotetext{
6 The name "FOS4" originated when it was a target for the HST Faint Object Spectrograph in the 1990s (Davidson et al. 1995; Humphreys et al. 1999; Zethson et al. 1999). Apart from small pointing differences, FOS4 is the same as the "Center of SE Lobe" in Figure 2(c) of Humphreys et al. and Figure 3(c) of Zethson et al. It was further discussed by Smith et al. (2003), Weis et al. (2005), and Stahl et al. (2005). The 1991-1997 HST FOS data may be worth re-examining today for comparison with later STIS observations.
} 
Table 1

HST Photometry Results ${ }^{\mathrm{a}}$

\begin{tabular}{|c|c|c|c|}
\hline MJD & Year & $\begin{array}{c}\text { Flux Density } \\
\left(10^{-11} \mathrm{erg} \mathrm{cm}^{-2} \mathrm{~s}^{-1} \AA^{-1}\right)\end{array}$ & $\begin{array}{l}\text { STMAG }^{\mathrm{b}} \\
\text { (mag) }\end{array}$ \\
\hline \multicolumn{4}{|c|}{ WFPC2 PC/F255W Filter } \\
\hline 54717.1 & 2008.69 & 1.074 & 6.323 \\
\hline 54787.8 & 2008.88 & $1.126 \pm 0.013$ & $6.271 \pm 0.013$ \\
\hline 54807.4 & 2008.93 & $1.099 \pm 0.035$ & $6.299 \pm 0.035$ \\
\hline 54834.0 & 2009.01 & $1.189 \pm 0.004$ & $6.212 \pm 0.004$ \\
\hline 54838.4 & 2009.02 & $0.967 \pm 0.014$ & $6.437 \pm 0.016$ \\
\hline 54841.5 & 2009.03 & $0.806 \pm 0.006$ & $6.635 \pm 0.008$ \\
\hline 54845.5 & 2009.04 & $0.598 \pm 0.017$ & $6.959 \pm 0.031$ \\
\hline 54854.5 & 2009.06 & $0.451 \pm 0.003$ & $7.265 \pm 0.008$ \\
\hline 54872.4 & 2009.11 & $0.437 \pm 0.019$ & $7.298 \pm 0.048$ \\
\hline 54949.2 & 2009.32 & $0.937 \pm 0.011$ & $6.471 \pm 0.013$ \\
\hline \multicolumn{4}{|c|}{ WFPC2 PC/F336W Filter } \\
\hline 54717.1 & 2008.69 & $1.108 \pm 0.035$ & $6.289 \pm 0.034$ \\
\hline 54787.8 & 2008.88 & $1.114 \pm 0.041$ & $6.283 \pm 0.040$ \\
\hline 54807.4 & 2008.93 & $1.142 \pm 0.031$ & $6.256 \pm 0.030$ \\
\hline 54834.0 & 2009.01 & $1.351 \pm 0.005$ & $6.073 \pm 0.004$ \\
\hline 54838.4 & 2009.02 & $1.399 \pm 0.037$ & $6.036 \pm 0.029$ \\
\hline 54841.5 & 2009.03 & $1.388 \pm 0.023$ & $6.045 \pm 0.018$ \\
\hline 54845.5 & 2009.04 & $1.300 \pm 0.007$ & $6.116 \pm 0.006$ \\
\hline 54854.5 & 2009.06 & $1.004 \pm 0.020$ & $6.396 \pm 0.022$ \\
\hline 54872.4 & 2009.11 & $1.008 \pm 0.027$ & $6.392 \pm 0.029$ \\
\hline 54949.2 & 2009.32 & $1.599 \pm 0.009$ & $5.890 \pm 0.006$ \\
\hline \multicolumn{4}{|c|}{ STIS Synthetic/F250W Filter } \\
\hline 55062.0 & 2009.63 & & 6.12 \\
\hline 55258.7 & 2010.17 & & 5.89 \\
\hline 55428.3 & 2010.63 & & 5.65 \\
\hline \multicolumn{4}{|c|}{ STIS Synthetic/F330W Filter } \\
\hline 55062.0 & 2009.63 & & 5.63 \\
\hline 55258.7 & 2010.17 & & 5.55 \\
\hline 55428.3 & 2010.63 & & 5.35 \\
\hline
\end{tabular}

Notes.

a Earlier results can be found in Martin \& Koppelman (2004) and Martin et al. (2006b, 2010).

b The STMAG photometric system is calibrated for direct comparison of fluxes with similar filters in different instruments.

usually did not allow observations with a vertical slit orientation ("the parallactic angle"). Therefore, in each case we calculated the apparent position of the star as a function of wavelength and used the offset slit position that was closest to the star for blue wavelengths. This procedure was adequate because, in effect, the slit positions overlapped due to the combination of slit width and seeing; we did not attempt to measure accurate absolute fluxes. For more information see Technical Memo 14 on the $\eta$ Car Treasury Program Web site. ${ }^{7}$

We prepared two-dimensional spectrograms using the standard GMOS data reduction pipeline in the Gemini IRAF package and extracted one-dimensional spectra via a routine developed earlier for use with HST STIS (Martin et al. 2006a). At each wavelength our software integrates the counts along a line perpendicular to the dispersion, weighted by a mesa-shaped function centered on the local spectral trace. We used a mesa function with base width $=11$ pixels and top width $=7$ pixels, about 0.8 and $0^{\prime \prime} .5$, respectively. The seeing was roughly $0.5-11^{\prime \prime} .5$, so each GMOS spectrum discussed represents a region about $1^{\prime \prime}$ across. The spectra were rectified using a LOESS fit (Cleveland 1979; Cleveland \& Devlin 1988). The pipeline wavelength calibration

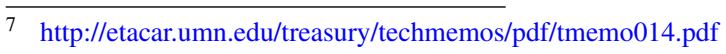

Table 2

Gemini GMOS Journal $^{\mathrm{a}}$

\begin{tabular}{|c|c|c|c|c|c|c|}
\hline Name $^{b}$ & Date & MJD & Phase & $\begin{array}{c}\text { Cenwave } \\
(\lambda)\end{array}$ & $\begin{array}{c}\text { Star }^{\mathrm{c}} \\
\text { Exp. Time } \\
\text { (s) }\end{array}$ & $\begin{array}{c}\text { FOS }^{\text {d }} \\
\text { Exp. Time } \\
\text { (s) }\end{array}$ \\
\hline $\mathrm{gH} 45$ & 2007 Jun 16 & 54267.1 & 1.707 & 4300 & 10 & $\ldots$ \\
\hline $\mathrm{gH} 45$ & 2007 Jun 17 & 54269.0 & 1.708 & 4300 & 10 & $\ldots$ \\
\hline $\mathrm{gH} 49$ & 2007 Jun 30 & 54281.0 & 1.714 & 5600 & 40 & 220 \\
\hline gI11 & 2008 Feb 11 & 54507.4 & 1.826 & 4300 & 77 & 377 \\
\hline gI11 & 2008 Feb 13 & 54509.2 & 1.827 & 5600 & 4 & 260 \\
\hline gI50 & 2008 Jul 5 & 54652.0 & 1.897 & 5600 & 40 & 260 \\
\hline gI54 & 2008 Jul 17 & 54665.0 & 1.904 & 4300 & 10 & 300 \\
\hline gI85 & 2008 Nov 8 & 54778.3 & 1.960 & 5200 & 40 & 453 \\
\hline gI85 & 2008 Nov 8 & 54778.3 & 1.960 & 4300 & 77 & 377 \\
\hline gI90 & 2008 Nov 27 & 54797.3 & 1.969 & 5200 & 40 & 450 \\
\hline gI90 & 2008 Nov 27 & 54797.3 & 1.969 & 4300 & 77 & 377 \\
\hline gI96 & 2008 Dec 18 & 54818.3 & 1.979 & 5200 & 40 & 450 \\
\hline gI96 & 2008 Dec 18 & 54818.4 & 1.979 & 4300 & 7 & 377 \\
\hline gI98 & 2008 Dec 25 & 54825.3 & 1.983 & 5200 & 40 & 450 \\
\hline gI98 & 2008 Dec 25 & 54825.4 & 1.983 & 4300 & 77 & 377 \\
\hline gI99 & 2008 Dec 31 & 54831.3 & 1.986 & 5200 & 40 & 450 \\
\hline gI99 & 2008 Dec 31 & 54831.4 & 1.986 & 4300 & 10 & 70 \\
\hline gJ01 & 2009 Jan 4 & 54835.3 & 1.988 & 5200 & 45 & 450 \\
\hline gJ01 & 2009 Jan 4 & 54835.3 & 1.988 & 4300 & 55 & 377 \\
\hline gJ02 & 2009 Jan 9 & 54840.2 & 1.990 & 5200 & 45 & 450 \\
\hline gJ02 & 2009 Jan 9 & 54840.2 & 1.990 & 4300 & 33 & 377 \\
\hline gJ03 & 2009 Jan 12 & 54843.3 & 1.992 & 5200 & 45 & 450 \\
\hline gJ03 & 2009 Jan 12 & 54843.3 & 1.992 & 4300 & 6 & 377 \\
\hline gJ04 & 2009 Jan 15 & 54846.2 & 1.993 & 5200 & 15 & 450 \\
\hline gJ04 & 2009 Jan 15 & 54846.2 & 1.993 & 4300 & 33 & 377 \\
\hline gJ05 & 2009 Jan 21 & 54852.3 & 1.996 & 5200 & 4 & 450 \\
\hline gJ05 & 2009 Jan 21 & 54852.3 & 1.996 & 4300 & 6 & 300 \\
\hline gJ06 & 2009 Jan 24 & 54855.3 & 1.998 & 5200 & 4 & 260 \\
\hline gJ06 & 2009 Jan 24 & 54855.4 & 1.998 & 4300 & 6 & 300 \\
\hline gJ07 & 2009 Jan 29 & 54860.4 & 2.000 & 5200 & 9 & 450 \\
\hline gJ07 & 2009 Jan 29 & 54860.4 & 2.000 & 4300 & 33 & 377 \\
\hline gJ09 & 2009 Feb 5 & 54867.2 & 2.004 & 5200 & 15 & 450 \\
\hline gJ09 & 2009 Feb 5 & 54867.3 & 2.004 & 4300 & 6 & 300 \\
\hline $\mathrm{gJ} 13$ & 2009 Feb 19 & 54881.2 & 2.010 & 5200 & 4 & 450 \\
\hline $\mathrm{gJ} 13$ & 2009 Feb 19 & 54881.3 & 2.011 & 4300 & 6 & 300 \\
\hline $\mathrm{gJ} 20$ & 2009 Mar 17 & 54907.3 & 2.023 & 5200 & 4 & $\ldots$ \\
\hline $\mathrm{gJ} 20$ & 2009 Mar 17 & 54907.3 & 2.023 & 4300 & 6 & $\ldots$ \\
\hline $\mathrm{gJ} 32$ & 2009 Apr 28 & 54949.1 & 2.044 & 5200 & 4 & 260 \\
\hline gJ32 & 2009 Apr 28 & 54949.1 & 2.044 & 4300 & 6 & 300 \\
\hline gJ56 & 2009 Jul 23 & 55036.0 & 2.087 & 5200 & 4 & 260 \\
\hline gJ56 & 2009 Jul 24 & 55036.0 & 2.087 & 4300 & 77 & 300 \\
\hline $\mathrm{gK} 02$ & 2010 Jan 8 & 55204.32 & 2.170 & 5200 & 4 & $\ldots$ \\
\hline gK02 & 2010 Jan 8 & 55204.34 & 2.170 & 4300 & 4 & $\ldots$ \\
\hline gK05 & 2010 Jan 20 & 55216.29 & 2.176 & 5200 & $\ldots$ & 260 \\
\hline
\end{tabular}

Notes.

${ }^{\mathrm{a}}$ P.A. $=160^{\circ}$, grating: B1200_G5321.

b As listed in http://etacar.umn.edu/.

c Include slit positions less than \pm 0 '.375 from the star at $\lambda 4687$.

d Include slit positions $-1^{\prime \prime}$ to $-3^{\prime \prime}$ from the star at $\lambda 4687$.

e Several exposures were taken on each data, combined exposure times are listed.

was improved using the interstellar Ca II $\mathrm{K}$ absorption line at $\lambda 3935$ and the interstellar absorption line at $\lambda 5782$. The absolute wavelength scale was obtained with HST STIS spectra that have better wavelength calibrations. Throughout this paper, we quote vacuum wavelengths and heliocentric Doppler velocities.

\subsection{Concerning Times and Phases}

When referring to "phase" in the 5.5 year cycle, we consistently use the Treasury Project system with period 2023.0 
days, which has been employed since 2003 without any need for revision; see http://etacar.umn.edu/, comments at the end of Section 2 in Mehner et al. (2010a), and an Appendix to this paper. We denote time within a spectroscopic event by $t$, such that $t=0$ at MJD 54860.0 (2009 January 29), MJD 52837.0 (2003 July 17), etc. Periastron most likely occurs within the range $t \approx-15$ to +15 days. Teodoro et al. (2011) arbitrarily use a different zero point that corresponds to $t \approx-18$ days, but the rationale for the long-standing Treasury Project system is noted in the Appendix.

\section{LIGHT CURVE OF THE STAR 2008-2011}

The light curve of the central star from HST data has two crucial advantages that have been overlooked in some discussions of $\eta$ Car's photometric record. First, all ground-based photometry includes ejecta at radii $r>0$ '. 15 , practically unrelated to short-term variations of the central star. This contamination is remarkably strong, varies with time differently than the star, and is difficult to quantify. In recent years, the ejecta accounted for $40 \%-70 \%$ of the brightness in the best ground-based photometry at visual wavelengths; see Figure 3 in Martin et al. (2006b). Since this relative fraction has lately been decreasing on a timescale of $\sim 10$ years (see the reference just cited), future ground-based photometry may eventually become representative of the star but pre-2005 measurements were dominated by ejecta. The contamination was probably still important during the 2009 event, but the amount is unclear; see Mehner et al. (2010b) and the discussion below. Fortunately, the high spatial resolution of HST allows us to sample the central object itself, $r<0$ '. 15 , with little extraneous contamination-albeit with less precision than high-quality groundbased photometry (cf. Fernández-Lajús et al. 2009, 2010 and http://etacar.fcaglp.unlp.edu.ar/). A second obvious and important advantage of $H S T$ is access to the UV region $\lambda<300 \mathrm{~nm}$, where the largest photometric changes occur during a spectroscopic event (see references in Section 2.1).

Indeed the HST UV data illustrate an important fact that is not obvious in the ground-based photometry: $\eta$ Car's 2009.0 event differed from its 2003.5 predecessor. Figure 1 shows the light curve in the F250W and F330W filters from 1998 through 2010 based on HST ACS/HRC and WFPC2 images and STIS spectra. The two smaller panels expand the light curve around the time of the event and show the minima for the 2009.0 and 2003.5 events superimposed. The depth of the minimum in the 2009 event was about $1.1 \mathrm{mag}$ in the F250W filter, which is sensitive to the Fe II forest, and 0.4 mag in the F330W filter. By contrast, the corresponding brightnesses decreased only half as much in 2003. It is clear from the timing of the observations that this difference is not simply due to a missed minimum in 2003.5. In order to have a 1 mag amplitude undetected in the 2003.5 observations, the $250 \mathrm{~nm}$ brightness would need either a strong negative "spike" only a few days long or an appreciably delayed minimum in terms of phase in the 5.5 year cycle, or both. Either of these possibilities would be as remarkable and unexpected as a major difference in amplitudes. (Unfortunately the minimum near 1998.0 is almost unknown.)

Fernández-Lajús et al. (2010) observed a similar but much less dramatic difference between events at visual wavelengths. In 2008-2009, they observed decreases of 0.15-0.25 mag in the UBVRI bands, only $0.02-0.03$ mag deeper than during the 2003.5 event. It is unclear whether the difference in the depth of the minimum was small because they observed longer wavelengths, or because they measured the brightness of star
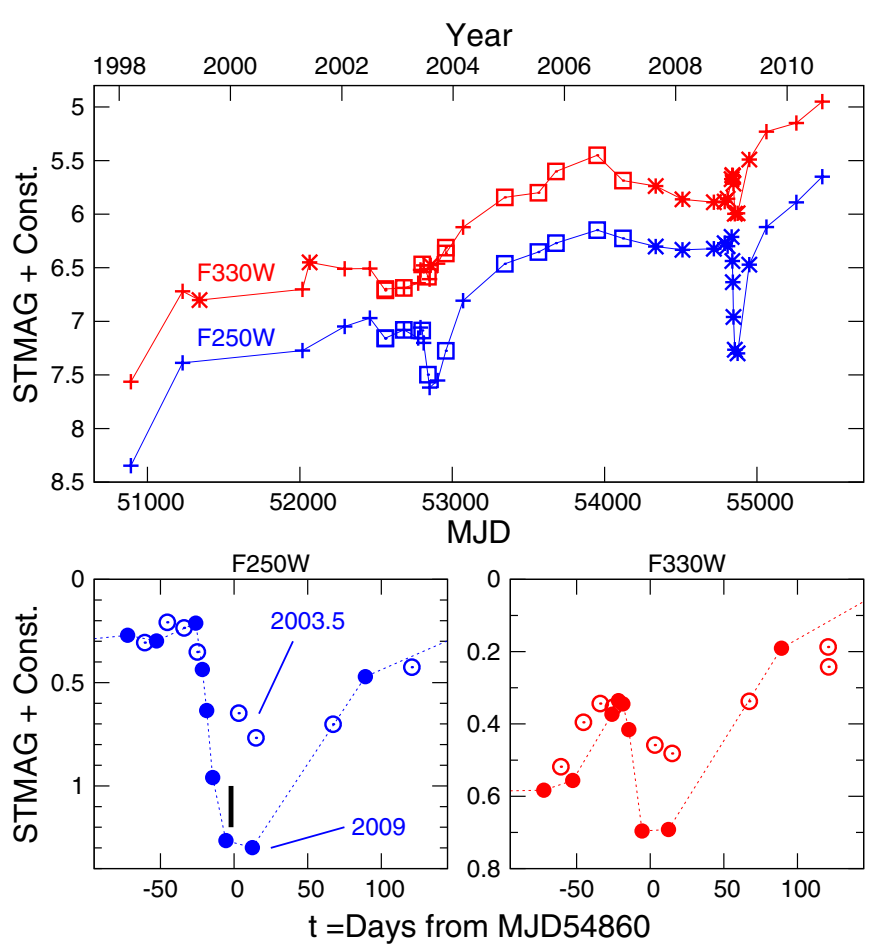

Figure 1. HST UV photometry. The upper panel shows the light curve from 1998 to 2011 (boxes: ACS/HRC, stars: WFPC2, crosses: STIS/CCD, values for F330W are shifted by $-0.4 \mathrm{mag}$ ). The two lower panels compare F250W and F330W brightness variations during the 2009.0 spectroscopic event (filled circles and dotted line) with the 2003.5 spectroscopic event (open circles). Day " 0 " corresponds to MJD 52837 for the 2003.5 event and MJD 54860 for the 2009.0 event. The black mark in the lower left panel indicates the date of the $V$ band minimum observed by Fernández-Lajús et al. (2010).

(A color version of this figure is available in the online journal.)

plus inner ejecta rather than the star alone. Unfortunately, we could not obtain suitable HST data at blue to red wavelengths in 2005-2009, because even the shortest integration time allowed for WFPC2 would have greatly overexposed the star with any of the filters appropriate for photometry at $\lambda>360 \mathrm{~nm}$.

The ground-based visual light curves suggest a minimum in 2009 between $t=-2$ days and +3 days (FernándezLajús et al. 2010, observations at MJD 54858-54863; $t$ was defined in Section 2.3 above). In HST data, observations at $t=-5$ days and +12 days showed the lowest brightness, in agreement with their data. Other aspects of the 2009 event are consistent with broadband optical and infrared observations of past events (Fernández-Lajús et al. 2003, 2010; van Genderen et al. 2003; Whitelock et al. 2004). The star began brightening at an accelerated rate about 40-50 days prior to the onset of the event. It brightened about $0.1 \mathrm{mag}$ in F250W and 0.2 mag in F330W before it steeply declined. Then, after about 150 days in F250W and about 100 days in F330W, the star returned to pre-event levels and resumed its long-term brightening trend.

Why have the events become successively deeper in UV photometry (Figure 1)? Here is one line of reasoning. The primary star's ill-defined UV photosphere is located in the opaque wind, far outside the star's surface. If the wind density is gradually decreasing on a timescale of $\sim 10$ years (Section 1 above; Mehner et al. 2010b; Martin et al. 2006b), then the photosphere seen at most times must be shrinking and becoming hotter. During a spectroscopic event, however, the mass-loss rate may be enhanced, at least at low latitudes. This possibility depends on the secondary star's tidal/radiative influence and 
on the size of the primary wind's acceleration zone (Davidson 1997, 1999; Hillier et al. 2001; Smith et al. 2003; Martin et al. 2006a). During an event the UV photosphere may therefore depend on different parameters than it does between events. (In principle, near periastron the secondary star might even be able to induce an outflow that is unrelated to the primary's usual wind. We do not advocate such an extreme model here, but it illustrates the basic idea.) Meanwhile, the Fe II forest around $\lambda \sim 260 \mathrm{~nm}$ (Davidson et al. 1999b; Hillier et al. 2001) is sensitive to temperature and density. For these reasons, one should not be surprised if the UV photospheric size, temperature, and brightness during events follow a different long-term trend than they do at other phases of the cycle (Figure 1). The same considerations apply to latitude and longitude dependences of the inner wind, even if there is no enhancement of total mass-loss rate (Smith et al. 2003).

\section{He II $\lambda 4687$ AND X-RAYS}

He II $\lambda 4687$ provides extraordinary clues to the nature of the spectroscopic event. This is by far the highest-excitation feature known in $\eta$ Car's UV-to-IR wind spectrum, it appears only briefly at a certain stage in each event, and it probably signals a flood of very soft X-rays. Steiner \& Damineli (2004) first drew attention to it, but Martin et al. (2006a) disagreed with their flux measurements and interpretation, and Teodoro et al. (2011) concur with the latter in most respects. Based on the Martin et al. analysis, we can summarize the relevant physics.

1. Since the observed feature almost certainly results from $\mathrm{He}^{++} \rightarrow \mathrm{He}^{+}$recombination, a temporary source of $\mathrm{He}^{+}-$ ionizing photons $(h v>54 \mathrm{eV})$ is required. Shocked gas flowing through the wind-wind collision zone does not produce enough $\lambda 4687$ emission via normal cooling.

2. Nearly all authors agree that the two stars produce very little radiation above $54 \mathrm{eV}$. Therefore, the relevant photons are most likely 54-500 eV X-rays produced in the wind-wind shock structure. Shocked gas of the primary wind, with preshock velocities below $600 \mathrm{~km} \mathrm{~s}^{-1}$, is favorable for creating soft X-rays.

3. The most suitable locale for $\lambda 4687$ emission is in the primary wind just before it encounters the colliding-wind shocks, and/or in locally cooled condensations within the shocked region. Since the primary-wind shock is unstable (Pittard \& Corcoran 2002; Soker 2003), these two choices may co-exist in roughly the same large-scale volume if one smooths over the complex small-scale structures. In either case $\lambda 4687$ is excited via photoionization by the soft $\mathrm{X}$-rays mentioned above.

4. The most plausible energy source is the primary wind. A naive assessment predicts that the soft X-rays are inadequate to explain $\lambda 4687$ by a factor of $3-10$, but several effects improve the efficiency. Martin et al. described radiative excitation effects that amplify $\lambda 4687$; instabilities in the shocked region tend to increase the number of very soft $\mathrm{X}$-ray photons; and a brief rise in the primary-wind outflow (hinted by other observations) would also help. With reasonable parameters, these details can enhance the He II $\lambda 4687$ flux by a sufficient factor.

5. The supply of soft X-rays can temporarily rise to very high levels if the fast secondary-wind shock becomes unstable like the primary-wind side. In that case the entire wind-wind interface "disintegrates" and "collapses," and a chaotic ensemble of subshocks and oblique shocks may exist for a few days or weeks. This phenomenon may explain the brevity of the $\lambda 4687$ flash as well as the disappearance of 2-10 keV X-rays.

Some of these statements are controversial, but no other quantitative analysis has been published. Steiner \& Damineli (2004) proposed that He II emission occurs in the acceleration zone of the secondary wind, a much smaller region than those mentioned above. If one employs consistent physical parameters, their model predicts a $\lambda 4687$ flux two or three orders of magnitude too weak (Martin et al. 2006a; Soker \& Behar 2006). Soker \& Behar (2006) also focused on the inner wind of the secondary star, but their scenario was very different, emphasizing a collapse of the shock structure followed by accretion onto the secondary. They gave qualitative arguments for enhanced $\lambda 4687$ emission in specified circumstances, but did not quantify the excitation physics. Their model includes some appealing components which we note in later sections below. Teodoro et al. (2011) recently indicated agreement with most of the above outline and did not attempt a new theoretical investigation. In summary, the Martin et al. assessment is still the only detailed account of the radiative processes; and so far there have been no strong arguments against it apart from geometrical details. Below we shall mention various agreements between authors, and then some crucial disagreements.

\subsection{The First He II Maximum During the 2009.0 Event}

Figure 2 shows a time sequence of the He II $\lambda 4687$ profile during the 2009.0 event, based on Gemini GMOS data. One can liken its appearance to a wave that first moves leftward in the figure and then is reflected back toward the right. (Note the reversed asymmetry of the profile at $t=+21$ days versus -20 days. Here "reflection" alludes to the line profile, not a real physical reflection.) The greatest source of uncertainty in this feature's strength is the underlying continuum level, which, following Martin et al. (2006a), we estimate by interpolation between 4605 and $4744 \AA^{8}{ }^{8}$

Observations reported by Teodoro et al. (2011) agree well with ours. At its maximum, the He II $\lambda 4687$ emission extended across $20 \AA$ or more $\left(\Delta V>1200 \mathrm{~km} \mathrm{~s}^{-1}\right)$ and had a much larger flux than one might guess from the apparent size of the line profile. This is true because the apparent "continuum" on each side of the line includes considerable $\lambda 4687$ emission; see Figure 3 in Martin et al., Figure 2 in Teodoro et al., and the marks on the right side of our Figure 2. Part of the $20 \AA$ line width may conceivably be due to Thomson scattering rather than bulk velocities. Here we shall ignore some minor anomalies, e.g., the profile seen at $t=-35$ days was blueshifted less than either of its neighbors at -42 and -29 days.

Figure 3 shows the time development of equivalent width and Doppler velocity for He II $\lambda 4687$ in our Gemini data. Here "equivalent width" refers to flux between 4675 and $4694 \AA$ $\left(-770\right.$ to $\left.+450 \mathrm{~km} \mathrm{~s}^{-1}\right)$; other spectral features hide the farther line wings. Our equivalent width measurements are listed in Table 3. "Velocity" in Figure 3 refers to the line's peak. As Teodoro et al. have noted, the main, double-peaked, 40 day $\lambda 4687$ flash in 2009 closely resembled the 2003.5 event. Its flux grew concurrently with the decline of $2-10 \mathrm{keV}$ X-rays (Section 4.3 below); the maximum equivalent width agreed

\footnotetext{
8 Steiner \& Damineli (2004) greatly underestimated the peak $\lambda 4687$ luminosity in 2003 because they chose too high a continuum level, shown in Figure 3 in Martin et al. (2006a). Teodoro et al. (2011) later adopted the Martin et al. method.
} 


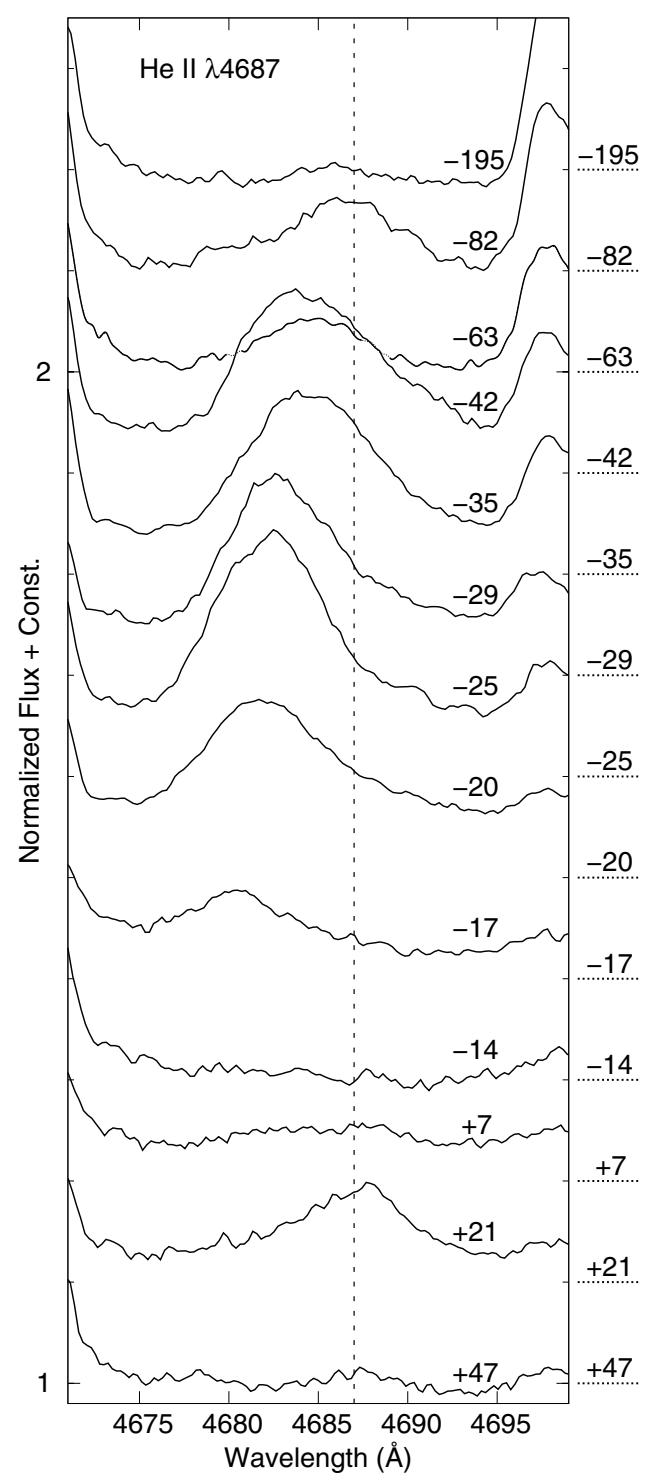

Figure 2. Time sequence of the He II $\lambda 4687$ emission during the 2009 event in Gemini GMOS observations. Continuum was normalized to unity at $\lambda 4740$ and is indicated with horizontal dotted lines on the right side. Offset between tracings is 0.1 . Number of days before (-) and after (+) MJD 54860 are indicated next to each spectrum. The dotted vertical line indicates the position of He II $\lambda 4687$ at zero radial velocity.

with the Martin et al. value for 2003.5 within measurement uncertainties; the time of significant brightness extended over 4-6 weeks; and the decline occurred in only one week.

The most precise time marker for $\lambda 4687$ emission is the midpoint of its decline, which occurred at MJD 54843 or $t=-17$ days. The corresponding time in 2003 was close to MJD 52821 (Steiner \& Damineli 2004), and the difference of $2022 \pm 2$ days matches the consensus 2023 day spectroscopic period (see the Appendix). The decline midpoint in our HST photometry at $\lambda \sim 250 \mathrm{~nm}$ occurred within a day or two of the same time (Section 3). Moreover, within measurement errors, the most negative $\lambda 4687$ Doppler velocity also coincided with the flux decline midpoint. (Steiner \& Damineli noticed the same coincidence in 2003 and considered it evidence for an eclipse.) At the time of maximum $\lambda 4687$ brightness 8 days earlier, the line peak had $V_{\text {doppler }} \approx-310 \mathrm{~km} \mathrm{~s}^{-1}$ (heliocentric); but then it rapidly moved to $-420 \mathrm{~km} \mathrm{~s}^{-1}$ at the decline midpoint. Later

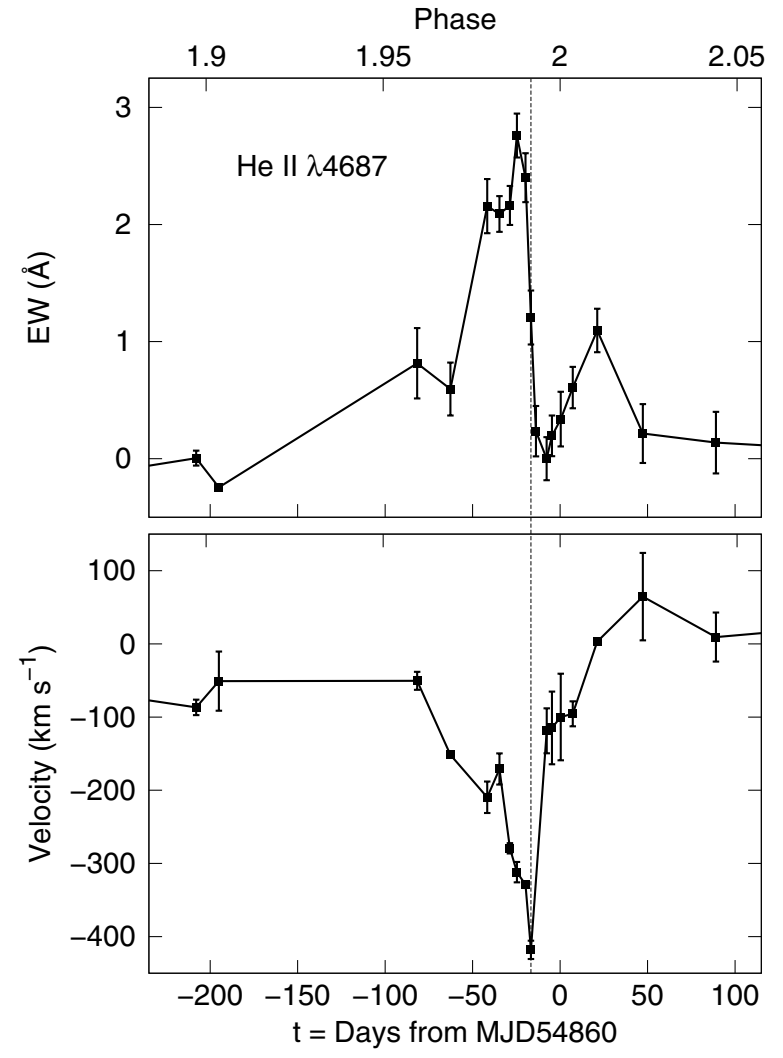

Figure 3. Equivalent width and radial velocity measurements of the $\mathrm{He}$ II $\lambda 4687$ emission on the star during the 2009 event in Gemini GMOS data. The dotted vertical line indicates the time of maximum negative radial velocity which occurs at the flux decline midpoint. This is also true for previous events (Steiner \& Damineli 2004).

Table 3

Equivalent Width and Radial Velocity of He II $\lambda 4687$ in Gemini GMOS Data on the Star

\begin{tabular}{llllrc}
\hline \hline Name $^{\mathrm{a}}$ & \multicolumn{1}{c}{$\begin{array}{c}\text { Date } \\
(\mathrm{UT})\end{array}$} & MJD & Phase & $\begin{array}{c}\text { EW } \\
(\AA)\end{array}$ & $\begin{array}{c}V_{\text {rad }}^{b} \\
\left(\mathrm{~km} \mathrm{~s}^{-1}\right)\end{array}$ \\
\hline gH45 & 2007 Jun 16 & 54268.0 & 1.707 & $-0.25 \pm 0.02$ & $15.09 \pm 6.11$ \\
gH49 & 2007 Jun 30 & 54281.0 & 1.714 & $-0.19 \pm 0.01$ & $-39.60 \pm 46.88$ \\
gI11 & 2008 Feb 11 & 54507.4 & 1.826 & $-0.34 \pm 0.03$ & $-35.39 \pm 54.12$ \\
gI50 & 2008 Jul 5 & 54652.0 & 1.897 & $0.01 \pm 0.06$ & $-86.80 \pm 10.60$ \\
gI54 & 2008 Jul 17 & 54665.0 & 1.904 & $-0.25 \pm 0.01$ & $-50.83 \pm 40.44$ \\
gI85 & 2008 Nov 8 & 54778.3 & 1.960 & $0.82 \pm 0.30$ & $-50.46 \pm 12.29$ \\
gI90 & 2008 Nov 27 & 54797.3 & 1.969 & $0.60 \pm 0.23$ & $-151.68 \pm 2.73$ \\
gI96 & 2008 Dec 18 & 54818.3 & 1.979 & $2.16 \pm 0.23$ & $-209.59 \pm 21.47$ \\
gI98 & 2008 Dec 25 & 54825.3 & 1.983 & $2.09 \pm 0.15$ & $-170.93 \pm 21.29$ \\
gI99 & 2008 Dec 31 & 54831.3 & 1.986 & $2.16 \pm 0.17$ & $-279.30 \pm 7.06$ \\
gJ01 & 2009 Jan 4 & 54835.3 & 1.988 & $2.76 \pm 0.19$ & $-311.94 \pm 13.91$ \\
gJ02 & 2009 Jan 9 & 54840.2 & 1.990 & $2.40 \pm 0.21$ & $-328.94 \pm 4.58$ \\
gJ03 & 2009 Jan 12 & 54843.3 & 1.992 & $1.21 \pm 0.23$ & $-418.11 \pm 12.49$ \\
gJ04 & 2009 Jan 15 & 54846.2 & 1.993 & $0.24 \pm 0.22$ & $\ldots$ \\
gJ05 & 2009 Jan 21 & 54852.3 & 1.996 & $0.00 \pm 0.18$ & $-118.72 \pm 30.72$ \\
gJ06 & 2009 Jan 24 & 54855.3 & 1.998 & $0.20 \pm 0.17$ & $-114.85 \pm 49.71$ \\
gJ07 & 2009 Jan 29 & 54860.4 & 2.000 & $0.34 \pm 0.23$ & $-99.95 \pm 59.14$ \\
gJ09 & 2009 Feb 5 & 54867.2 & 2.004 & $0.61 \pm 0.18$ & $-95.55 \pm 17.06$ \\
gJ13 & 2009 Feb 19 & 54881.2 & 2.011 & $1.10 \pm 0.19$ & $3.08 \pm 3.97$ \\
gJ20 & 2009 Mar 17 & 54907.3 & 2.023 & $0.22 \pm 0.25$ & $64.57 \pm 59.78$ \\
gJ32 & 2009 Apr 28 & 54949.1 & 2.044 & $0.14 \pm 0.26$ & $9.28 \pm 33.48$ \\
gJ56 & 2009 Jul 24 & 55036.0 & 2.087 & $0.06 \pm 0.30$ & $27.39 \pm 19.36$ \\
gK02 & 2010 Jan 8 & 55204.3 & 2.170 & $0.08 \pm 0.19$ & $-56.88 \pm 7.80$ \\
\hline & & & & &
\end{tabular}

Note. ${ }^{a}$ As listed on the Eta Carinae Treasury Project site at http://etacar.umn. edu/. 


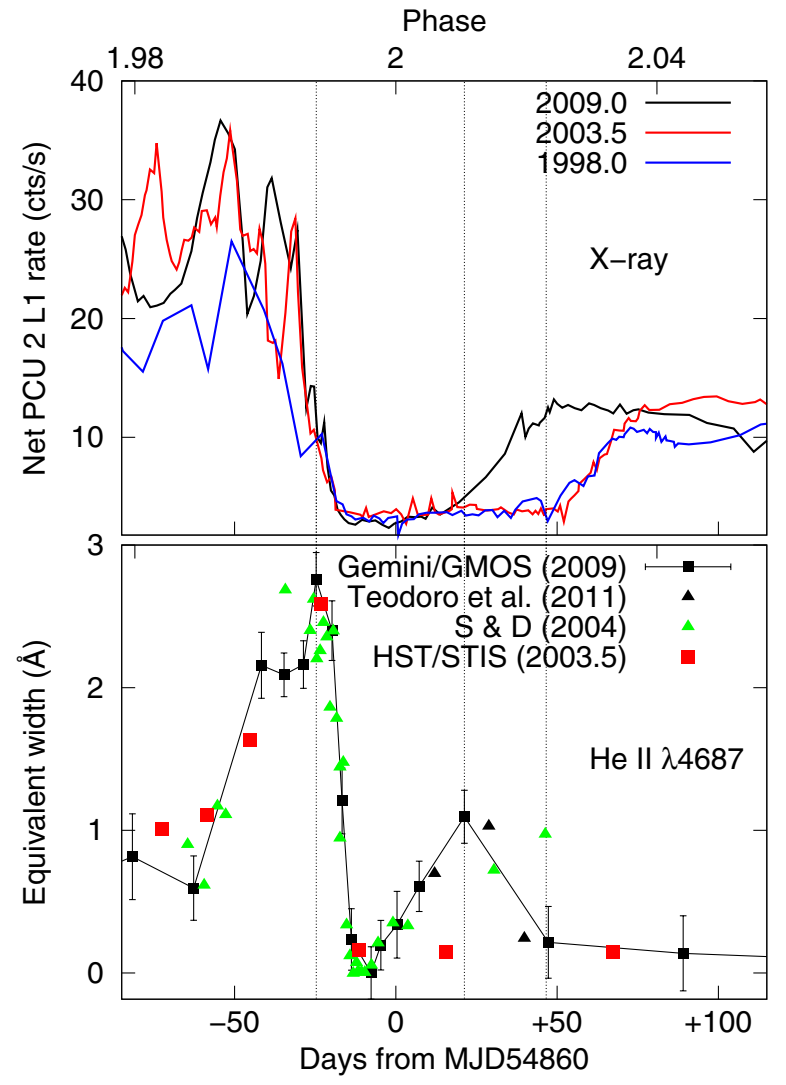

Figure 4. Top: X-ray light curve during the 1998, 2003.5, and 2009 events (from http://asd.gsfc.nasa.gov/Michael.Corcoran/eta_car/etacar_rxte_lightcurve/ index.html). Bottom: equivalent width of the He II $\lambda 4687$ emission during the 2009 event with Gemini GMOS (black squares) and selected data points (black triangles) from Teodoro et al. (2011) which constrain the timing of the second episode during the 2009 event further. The red squares are measurements of HST STIS data during the 2003.5 event. No significant He II emission was found in 2003 at $t \approx+20$ days in STIS data and measurements by Steiner \& Damineli (2004) during the 1992.5 and 1998 events (green triangles; these values are scaled by a factor of three, see footnote 8 ) indicate a delayed second episode. This casts doubt on the timing of the second episode found by Teodoro et al. (2011). Values for older events are shifted by multiples of 2023 days.

we shall indicate reasons why the similarity between the 2003 and 2009 records is somewhat surprising.

\subsection{The "Second Episode"}

A second, smaller He II $\lambda 4687$ flare occurred several weeks after the first, around $t \sim+20$ days in Figures 2 and 3. Its significance will appear in Section 4.3 below; it did not match the 2003 spectroscopic event. Independent of that question, two aspects of the "second He II episode" are noteworthy. First, the feature began to grow soon after its minimum at $t \sim-10$ days and continued to do so for about a month. Therefore, the emitting region was not entirely eclipsed by the primary wind. A second fact is the rapidity of change in Doppler velocity. The first maximum or episode attained $V_{\text {doppler }} \sim-420 \mathrm{~km} \mathrm{~s}^{-1}$, but the emission that reappeared several weeks later had $V_{\text {doppler }} \sim 0 \mathrm{~km} \mathrm{~s}^{-1}$. Meanwhile the line profile's asymmetry reversed as mentioned earlier (Figure 2). The overall velocity range exceeds the maximum projected orbital velocity variation for either star with any proposed 5.5 year orbit. On the other hand, the line-of-sight wind velocities and post-shock velocities can easily span a range of more than $400 \mathrm{~km} \mathrm{~s}^{-1}$ at various locations near the path followed by the secondary star near periastron. This statement may have interesting and

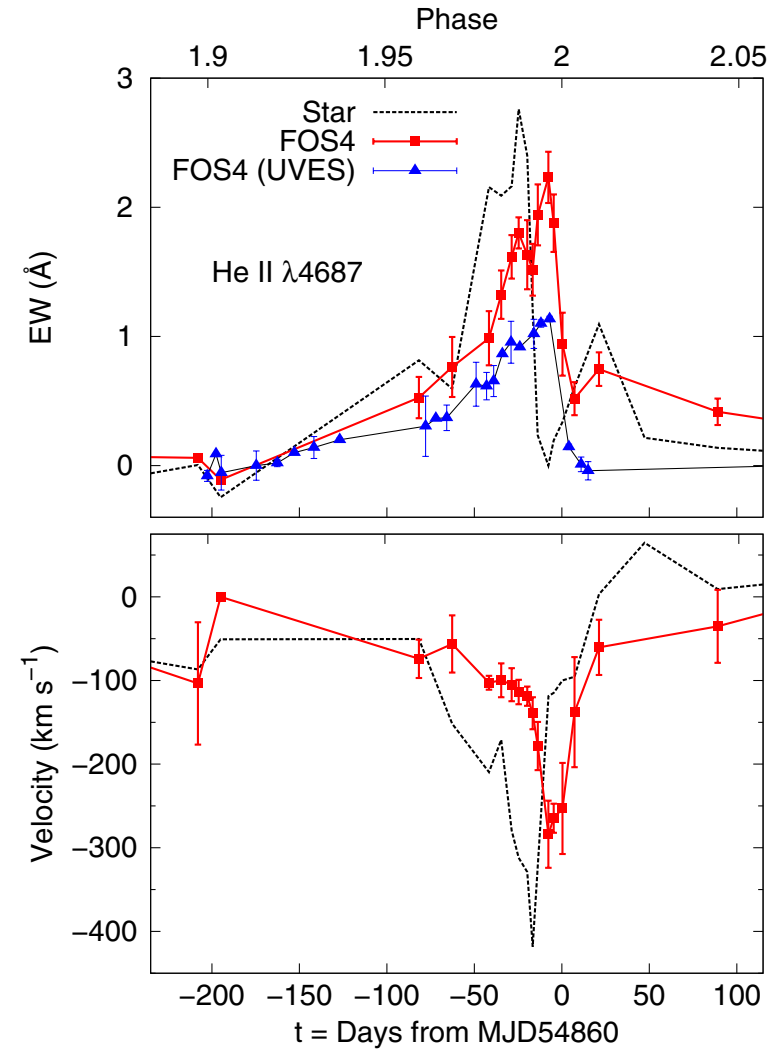

Figure 5. Equivalent width and radial velocity of He II $\lambda 4687$ at FOS 4 during the 2009 event observed with Gemini GMOS (red squares). Dashed curves are measurements on the star in direct view. The time delay between spectra of the star in direct view and spectra at FOS4 is about 18 days. Re-measurements of VLT UVES data during the 2003.5 event are consistent with this finding (blue triangles, values are shifted by 2023 days).

controversial implications for the orientation of the orbit, see Sections 4.4 and 6.

Unlike the first He II maximum, the second episode in 2009 apparently differed from the 2003.5 event. Here we disagree with Teodoro et al. about the 1992-2003 record, not 2009. The most reliable datum for this question was the HST STIS observation at MJD 52852 in 2003, carefully assessed by Martin et al. (2006a). Its timing corresponds to $t=+15$ days, only a few days before the phase of the second-episode peak seen in 2009. The 2003 STIS data showed no detectable line profile at that time, see Figure 5 in Martin et al. If one defines the emission feature as a bump or profile in the spectral tracing, then Martin et al. found a $3 \sigma$ upper limit of only $0.15 \AA$ for the equivalent width at MJD 52852. The points plotted in Figures 3-5 represent a different type of measurement, integrating a flux difference $f(\lambda)-f_{\mathrm{c}}(\lambda)$ over a $19 \AA$ interval. Here $f_{\mathrm{c}}(\lambda)$, the underlying continuum level, is the main source of error. The 2003 STIS data point at $t=+15$ days in Figure 4 may therefore be an overestimate, but the absence of a bump in the spectral tracing (see above) indicates that it is not likely to be a serious underestimate. The observation is consistent with equivalent width $(\mathrm{EW}) \sim$ zero at that time and excludes EW $\gtrsim 0.30 \AA$ A; see Sections 5 and 6.3 in Martin et al. If the 2003 event had a second He II episode matching the 2009 case, then the equivalent width at MJD 52852 should have been close to $0.9 \AA$, which would have been obvious in the STIS spectrum. Conclusion: the 2003 event did not include a second He II episode matching that seen in 2009. If one did occur at the same phase, then in 2003 it must have been much weaker than 2009, even though the first He II maximum was practically 
alike in both events. But there is no good evidence for such an occurrence, and in Section 4.3 we favor the simple view that either 2003 had no second episode or else it was delayed.

The ground-based data are extremely unclear on this question, especially since Teodoro et al. (2011) disagree with Steiner \& Damineli (2004) on major results even though they used similar, overlapping data sets. Only one data point is strongly pertinent: Teodoro et al. estimate $\operatorname{EW}(\lambda 4687) \approx 0.65 \pm 0.16 \AA$ at MJD 52841,11 days before the 2003 STIS observation noted above. In order to accept both this measurement and the STIS result, one would need to postulate a sudden decrease between them. This may be possible, but in that case the record would not resemble the 2009 second episode. A simpler explanation is that the MJD 52841 datum was an overestimate (see below). Teodoro et al. also note a data point with $\mathrm{EW} \approx 0.56 \AA$ at $\mathrm{MJD}$ 48825 in 1992 ( $t \approx+34$ days), and perhaps two weaker ones, but the doubts expressed below apply even more forcefully to them.

Here we must digress on comparisons between groundbased data and HST. All ground-based spectroscopy of $\eta$ Car is seriously (not just mildly) contaminated by emission-line ejecta at $r \sim 0^{\prime} .2-2^{\prime \prime}$ (Davidson et al. 1995). The size of this effect depends on the spectrograph aperture size and on variable atmospheric seeing. Moreover, it was worse in the 1990s because the star was relatively faint then (Martin et al. $2006 b)$. There is concrete evidence that narrow emission lines in the ejecta tend to mimic a weak "He II $\lambda 4687$ " feature when the data are smoothed. Details can be found in Mehner (2011); a brief summary follows. Gemini data and slightly decentered STIS spectra show many weak narrow spikes near $4687 \AA$. Most of them are scarcely distinguishable from noise, except that their wavelengths recur in independent observations. This fact is not surprising, since the ejecta are known to produce thousands of weak unidentified lines (Zethson 2001). When the spectra are heavily smoothed, some of these tiny features blend to produce a " $\lambda 4687$ " bump with height $\sim 0.5 \%-1 \%$ of the continuum level. This bump consistently appears near the same wavelength, and it closely resembles the feature reported by Steiner \& Damineli (2004) and Teodoro et al. (2011) in data from Pico dos Dias (OPD) at times when $\lambda 4687$ was weak. The OPD feature tends to be stronger by a factor of $\sim 2$, which can be ascribed to worse atmospheric seeing, a larger spectrograph aperture, and the fact that the apparent brightness ratio (ejecta/ star) was much larger in the 1990s than it is today (Martin et al. 2006b; Davidson et al. 1995). (Despite this long-term trend, however, Teodoro et al. found the feature in data as late as 2010.) In summary, the OPD data show symptoms of extraneous emission-line contamination, at a level that is not surprising but which affects most of the $\lambda 4687$ observations. HST STIS, on the other hand, had good enough spatial resolution to separate most of the ejecta. We conclude that pre-2004 ground-based data have very low weight compared to STIS in 2003 and many groundbased observations in 2009. Consequently, there is no significant evidence for a second He II $\lambda 4687$ flare around $t \sim+20$ days in 1992, 1998, and 2003.

There is an interesting puzzle in the observational record. If we assume similarity among the 1992, 1998, and 2003 spectroscopic events, then Steiner \& Damineli (2004) depicted a conspicuous second He II episode at a significantly delayed time $t$. Their two relevant OPD observations occurred at $t \sim+30$ days in 1998 and +50 days in 1992, plotted in Figure 4 as green triangles. These appeared to be stronger than the most doubtful cases mentioned above. If valid, they imply second He II flares in 1992 and 1998, but at phases roughly 30 days later than the 2009 case-which would make sense for reasons noted in Section 4.3 below. However, they contradict some 1992 ESO data listed by Teodoro et al. (2011), and they are not mentioned in the latter paper even though it includes other data from the same instrument. Either there were delayed second episodes in 1992 and/or 1998 (Steiner \& Damineli) or not (Teodoro et al.). These inconsistencies reinforce our opinion that ground-based spectroscopy of $\eta$ Car in those years was far less robust than the 2003 HST results and various observations in 2009.

In summary: one of the high-quality HST STIS observations in 2003 showed that the event in that year did not include a second He II episode matching the 2009 record; and this assertion is not contradicted by any reliable ground-based data. A weaker and/or later second episode may have occurred in 2003, as we discuss in Section 4.3.

\subsection{He II, X-Rays, and Shock Breakup}

Martin et al. (2006a) emphasized that He II $\lambda 4687$ was anticorrelated with $\eta$ Car's observable $2-10 \mathrm{keV}$ X-rays during the 2003.5 event; the $\lambda 4687$ flash occurred as the X-rays declined (Figure 4). This fact appeared very consistent with a shock breakup as outlined below, and the 2009 "second episode" now strengthens the case.

Davidson (2002) remarked that known shock instabilities, rather than the eclipse scenario that was popular at that time, can best explain the rapid disappearance of $\eta$ Car's 2-10 keV X-rays during a spectroscopic event. Soker (2003) noted quantitative details, Martin et al. (2006a) emphasized the relevance of He II $\lambda 4687$ to this concept (see Section 9.2 of that paper), and Soker \& Behar (2006) suggested an especially favorable mode of instability. Other researchers later adopted essentially the same ideas (Damineli et al. 2008a; Parkin et al. 2009; Teodoro et al. 2011). Two specific variants, with different causes but similar consequences, have been proposed.

1. A shock structure becomes unstable if radiative cooling exceeds expansion cooling (Stevens et al. 1992). The slow primary-wind shock of $\eta$ Car is very unstable in this regard, but the faster secondary-wind shock stabilizes the overall structure in calculated models (Pittard \& Corcoran 2002; Soker 2003). The secondary shock may become unstable near periastron, causing the entire shock structure to disintegrate on a timescale of 10-30 days (Section 9.2 in Martin et al. 2006a).

2. Soker \& Behar (2006) drew attention to another phenomenon that Stevens \& Kallman (1990) had studied for $\mathrm{X}$-ray binaries in general. Near periastron, soft X-rays from the shocked region can alter the ionization state of the secondary star's wind. A higher degree of ionization tends to weaken the line-driven acceleration, resulting in a slower wind speed ("radiative inhibition," a term later used by Parkin et al. 2009). This in itself would reduce the $2-10 \mathrm{keV}$ flux; but another consequence is that the balance of wind momenta is altered, pushing the shocks closer to the secondary star. In an extreme case the primary wind can entirely suppress the secondary wind.

Other instabilities certainly occur, such as Kelvin-Helmholtz which mixes gas from the two winds, and obvious thermal instability as shocked gas cools below $10^{6} \mathrm{~K}$, but the two processes listed above have mutually similar large-scale consequences and they might even develop together. Observable 2-10 keV X-rays rapidly and tremorously disappear as the highest temperature 
decreases, a flood of soft X-rays is created by the chaotic ensemble of local shocks as the overall structure breaks up, and the secondary wind may temporarily cease to exist. (Alternatively, it might survive in a slower form.) Martin et al. noted that empirically, without referring to physics, this view is supported by the growing unsteadiness of $2-10 \mathrm{keV}$ X-rays around their maximum.

Realistic simulation of these phenomena for $\eta$ Car would be extremely difficult, and many sets of parameters must be explored. Pending such models, one can estimate the relevant timescales from the overall size of the shocked region and local velocities within it: say 1-3 AU and 100-800 $\mathrm{km} \mathrm{s}^{-1}$, respectively. These values suggest $\Delta t \sim 2-50$ days, consistent with the observed behavior.

Shock disintegration or collapse was originally only one among several competing explanations for the $2-10 \mathrm{keV}$ X-ray crash during a spectroscopic event. Then, in 2003 the previously unrecognized He II $\lambda 4687$ feature behaved just as the shock breakup idea would have predicted. It appeared approximately when the hard X-rays peaked, it grew as they declined, and then ceased abruptly after a few weeks. As Martin et al. (2006a) noted, one would not expect this sequence and timing in an eclipse model like that of Pittard \& Corcoran (2002), for example.

Teodoro et al. (2011) have recently advocated a shock breakup model that closely resembles the earlier conclusions of Martin et al. We differ from Teodoro et al. in two important respects: first, they state that He II $\lambda 4687$ originates " $4-5$ AU downstream from the apex" within the shocked gas (p. 5). However, as implied in point 3 of the physics summary near the beginning of this section, there is no clear reason for this assumption. Wherever very soft X-rays occur, they indirectly create $\lambda 4687$ in the nearest suitable gas. Most of the applicable energy supply for soft X-rays near periastron is found at distances considerably less than $4 \mathrm{AU}$ from the pre-breakup apex or vertex. Moreover, it seems possible or even likely that soft X-rays and $\lambda 4687$ emission may continue for 5-20 days after the original largescale shock structure has ceased to exist. Our second difference is that Teodoro et al. give little attention to the evidentiary role of the unforeseen He II second episode in 2009; see below.

The 2009.0 event provides a valuable new clue, the He II second episode discussed in Section 4.2. Figure 4 shows that its behavior was qualitatively a reversal of the first episode seen 40 days earlier. The $\lambda 4687$ emission reappeared and peaked around $t \sim+20$ days while the $2-10 \mathrm{keV}$ X-rays were still weak; then it declined concurrently with the growth of the X-rays. The overall timescale was comparable to that seen in the first episode. In the shock-instability scenario, of course, we interpret this pattern as the re-formation of a large-scale shock structure when the relevant densities become sufficiently low for it to be quasi-stable. This view raises several questions.

1. As Figure 4 shows, the hard X-rays reappeared earlier than expected in 2009, around $t \sim+30$ days rather than $t \sim+60$ days as seen in 1998 and 2003 (Kashi \& Soker 2009a; Corcoran et al. 2010). Given this difference, should the earlier events have shown the same He II behavior pattern as 2009? The shock breakup hypothesis "predicts" He II $\lambda 4687$ flares around $t \sim+50$ days in 1998 and 2003, i.e., just before the hard X-rays reappeared. Equally important, a $\lambda 4687$ outburst as early as the 2009 case, $t \sim+20$ days, would not be expected. The second He II episodes would very likely have been weaker in those earlier events, because the $\lambda 4687$ production rate depends strongly on local densities. Consider, for example, a case with orbital eccentricity 0.9 and periastron at $t=0$, fairly consistent with most proposed orbit models. Then the separation between stars was about 3.5 AU during the pre-periastron $\lambda 4687$ maximum $(t \sim-20$ days $)$, but more than 6 AU at $t \approx+50$ days just before the X-rays reappeared in 1998 and 2003. If we assume an unchanged primary wind, these values imply a factor-of-three lower density at the expected time of reappearance. According to the Martin et al. analysis, this would reduce the $\lambda 4687$ flux by a factor of $3-10$ compared to its pre-periastron maximum-depending on local gas geometry, velocity gradients, etc. Moreover, the primary wind density may have been temporarily enhanced in the weeks before periastron (Davidson 1999; Martin et al. 2006a). One should therefore expect the second He II episode to have been weak in 1998 and 2003, possibly undetectable. In 2009, on the other hand, the second episode occurred fairly soon after periastron, so the relevant densities were probably comparable to those that had existed during the pre-periastron $\lambda 4687$ maximum. Indeed this distinction is the main reason why the 2009 record provides a valuable new clue. (Below we shall comment on why this happened so early.)

2. Is the observational record consistent with these expectations? The answer is "yes," but unfortunately the pre-2009 data were too sparse for confident conclusions. We noted in Section 4.2 that the second expectation, no substantial $\lambda 4687$ flare at $t \sim+20$ days in 2003 , is confirmed by STIS data. The question of a later flare, however, amounts to a conflict between observations reported by Steiner \& Damineli (2004) and those reviewed by Teodoro et al. (2011). The former data strongly imply a $\lambda 4687$ episode peaking around $t \sim+50$ days in 1992 and 1998, see Figure 4 and Section 4.2 above. Teodoro et al., on the other hand, omit those two data points and show no definite $\lambda 4687$ emission after $t \sim+35$ days in 1992 and 1998, and no data points in that time interval in 2003. Neither of these alternatives would contradict the shock breakup idea (see above), but it would be useful to know whether or not a detectable second episode occurred. Since the OPD data are not public (unlike HST and GMOS), it is difficult to assess these alternatives. Meanwhile, the 2009 data and 2003 STIS data constitute the only satisfying results on this question-but there were not enough STIS observations.

3. Why did the He II emission and hard X-rays reappear earlier than expected in 2009? Referring only to the X-rays, Kashi \& Soker (2009a) proposed an explanation based on a decrease in the primary star's wind. They had a specific model in mind, but their basic idea works for others as well. Since 1999 there have been hints that $\eta$ Car's wind density is (or was) becoming less dense on a timescale of $\sim 10$ years (e.g., Martin et al. 2006b; Davidson et al. 2005). Mehner et al. (2010b) recently reported strong spectroscopic evidence for such a change. Qualitatively, at least, one expects the wind-wind shock structure to recover sooner after periastron if the primary wind is less dense because in that case the worst instabilities are weakened. At first sight the 2009 He II second flare may seem paradoxical in this view, because, as noted above, lower densities tend to reduce the $\lambda 4687$ emission efficiency. A partial explanation is that the shock-region density at the time when it re-forms is not the same as the primary wind density measured at some fixed radius; the two stars were closer together at 
$t \sim+20$ days in 2009 than they were at $t \sim+50$ days in 2003. This brings us to the next question which also concerns timing and separation between the stars.

4. Suppose, as stated above, that the primary star's wind was less dense in 2008-2009. If so, why did not the shock structure survive later than expected before periastron? With reduced density $\rho_{\text {wind }}(r)$, one would expect the shocks to disintegrate at a smaller star-star separation, i.e., later. In fact the $2009 \mathrm{X}$-ray crash and the main He II $\lambda 4687$ flash occurred almost exactly 2023 days after the 2003 event (Section 4.1 and Figure 4). Perhaps this fact indicates that densities at the critical time did not represent the primary star's "normal" steady mass-loss rate. Instead, the inner wind may have been tidally or radiatively enhanced during the weeks before periastron, causing the relevant density to be approximately the same for each spectroscopic event. This hypothesis is not arbitrary or ad hoc, since it also helps to explain a photometric puzzle mentioned at the end of Section 3 above, and it was suggested long ago on other grounds (e.g., Davidson 1999; Martin et al. 2006a).

Teodoro et al. (2011) appear to concur with most, but not all, of the theoretical points that we have quoted from Martin et al. (2006a). One disagreement pertains to the He II "second episode" seen in 2009. Above we emphasized the anticorrelation between $\lambda 4687$ flux and hard X-rays at critical times. Teodoro et al., however, state instead that the two are correlated but $\lambda 4687$ is delayed by 16.5 days. In our opinion, semi-theoretical reasoning plus an empirical fact do not support that view. (1) Those authors interpret the 16 day delay as the time required for shocked gas to flow from a favorable X-ray region near the vertex or apex of the umbrella-shaped shock structure, to another location where it has cooled enough to produce He II emission. For this explanation, one needs a localized density enhancement, which must pass near the shock vertex and later reaches some particular radius at a well-defined time after flowing outward within the shocked zones, but these are unlikely assumptions in the context of an extended, chaotic, unstable shock structure. There is no clear reason for the localized density enhancement, only a tiny fraction of material flowing outward from either star passes close to the shock vertex, gas is vigorously mixed and diffused within the shock structure, the overall geometry is probably unstable at the critical time as noted above, and there is no reason to assume that He II emission occurs only beyond a certain distance from the vertex. In other words, the proposed rationale is unclear. (2) Our second, more empirical objection is simpler. The 16 day delay proposed by Teodoro et al. is obviously inconsistent with the $\lambda 4687$ second episode observed in 2009 (Figure 4). Maximum He II emission occurred after the hard X-ray peak in the first episode, but before the X-ray recovery in the second.

In summary: strictly speaking we cannot prove the shock breakup scenario, but the combined $\lambda 4687$ and X-ray data are impressively consistent with it. In terms of logic, the role of He II $\lambda 4687$ has been as follows. First, this feature was not recognized when the breakup idea was first proposed, but then it turned out to fit into that scenario in a remarkably natural way. Later, when a post-periastron "second He II episode" appeared in 2009 as discussed above, it too matched quite naturally, and the observed secular decrease in wind density provides a good explanation for the differences between 2003 and 2009. In neither case was there any need to alter the concept to fit new observations.

In terms of theoretical development, Davidson (2002) and Soker (2003) stressed that shock breakup is a reasonable con- cept for $\eta$ Car; then Martin et al. (2006a) and Soker \& Behar (2006) argued that it is a very likely one. Parkin et al. (2009) later cited other X-ray clues, notably that gas dynamic computations have not sustained the competing eclipse scenario. Given these facts, and lacking a viable alternative explanation, we conclude that $\eta$ Car's colliding-wind shock structure does, indeed, disintegrate and collapse during a spectroscopic event. Two separate questions are (1) whether the primary star has a mass-loss outburst at about the same time and (2) whether the secondary star accretes material then. See Section 6 below.

Is He II $\lambda 4687$ emission detectable in $\eta$ Car only during spectroscopic events? According to Steiner \& Damineli (2004) and Teodoro et al. (2011), this feature has been present at other times. On the other hand, Martin et al. (2006a) found no broad He II in non-event HST STIS spectra of the central star. STIS mapping data in 2009 June and December show no broad $\lambda 4687$ emission in extended regions around the star. Analyzing observations made with HST STIS, Gemini GMOS, and Irénée du Pont B \& C, we find that a weak emission feature exists but it is only about $3 \AA$ wide, i.e., narrower than the He II emission seen when it is bright. As noted in Section 4.2, it is most likely a blend of very weak, narrow, unidentified lines and not broad He II emission (Mehner 2011).

\subsection{He II $\lambda 4687$ in the Reflected Spectrum from the Pole (FOS4)}

Spectra reflected by dust in the Homunculus nebula give surprising new information about velocities in the 2009 event. The known geometry of the bipolar Homunculus allows us to correlate each position in the SE lobe with stellar latitude, assuming that the polar axis of $\eta$ Car is aligned with the Homunculus axis. FOS4 is a location near the center of the SE lobe (the one nearer to us) which reflects a nearly pole-on view of the stellar wind, $\sim 75^{\circ}$ latitude (Smith et al. 2003; Davidson et al. 1995; Zethson et al. 1999). Our direct view probably represents $\sim 45^{\circ}$ latitude (Davidson et al. 2001; Smith 2006). FOS4 is also useful for another reason, namely that groundbased spectra there are less contaminated by nebular lines than direct observations of the star are. (The reasons for this fact are not entirely clear, but generally speaking our direct view of the star appears to have more circumstellar extinction than the average line of sight.) FOS4 is located about 3".7 south and 2 ".5-3..5 east of the central star.

A spectrum reflected in the Homunculus has a light-traveltime delay $\Delta t$ corresponding to the additional path length, and an extra Doppler shift $\Delta V$ due to the "moving-mirror" effect (Meaburn et al. 1987; Stahl et al. 2005). With standard assumptions about the Homunculus, these are related by $\Delta V / c=\Delta t /$ (age), where "age" means elapsed time since the reflecting material was ejected in the 1840s. The value of $\Delta V$ depends on location in a straightforward, observable way that depends on the shape of the Homunculus lobe, see Figure 4 in Davidson et al. (2001). In fact we can define FOS4 as the location where $\Delta V=+100 \mathrm{~km} \mathrm{~s}^{-1}$, which implies $\Delta t \approx 20$ days for the 2003 and 2009 spectroscopic events. Stahl et al. (2005), however, reported a time delay of only 10 days for appearance and disappearance of He II $\lambda 4687$ in 2003, comparing VLT UVES observations of FOS4 to observations by Steiner \& Damineli (2004) which were centered on the star. If correct, this would cast doubt on standard analyses of the reflection model, Homunculus expansion, etc. The good time coverage of our Gemini GMOS data during the 2009 event, both on the 
star and on FOS4, allows us to re-evaluate this result. Figure 5 shows the equivalent width and radial velocity measurements of He II $\lambda 4687$ in spectra of the star in direct view and reflected at FOS4. It also includes re-measured equivalent widths in UVES data during the 2003.5 event, shifted by 2023 days. The GMOS data show that the time delay in the observed equivalent widths as well as radial velocities, between the direct view on the star and the pole-on view at FOS4, is about 18 days which confirms the expected $\Delta t \approx 20$ days within the attainable accuracy. Also, re-measurement of the UVES data during the 2003.5 event according to the method employed by Martin et al. (2006a) shows that the earlier data are in accordance with the GMOS 18 day value. We detected a positional gradient of $\Delta t$, which agreed with the simple model within the measurement errors. The geometry of the reflection process therefore appears satisfactory.

Unexpectedly, we find that the behavioral pattern of $\mathrm{He}$ II $\lambda 4687$ emission is very similar when viewed from different directions, i.e., in direct view of the star and reflected at FOS4, when we take the time delay into account. Values for the equivalent widths and radial velocities are slightly smaller at FOS4 than in the direct view. Figure 5 shows these results, with Doppler velocities corrected for the moving-mirror effect $\Delta V$. He I $\lambda 4714$ seems to exhibit similar behavior, but cannot be measured well because its line profile changes shape in a more complex way than He II $\lambda 4687$ (Figure 6; Section 5 below; Mehner 2011). These results suggest that velocities of helium lines are not simply related to orbital motion of the secondary star, if we assume that the orbit inclination is $i \sim$ $40^{\circ}-45^{\circ}$ like the Homunculus midplane, see references cited above. In standard models, the view from FOS4 should be almost perpendicular to the plane of the orbit, and therefore no large radial velocity variations should be observed there. Thus the data at FOS4 are very surprising.

As examples, let us mention just two of the published models. Soker \& Behar (2006) proposed that helium lines originate in the acceleration zone of the secondary star. Assuming we view the orbit at an inclination of $40^{\circ}-45^{\circ}$, then the He II Doppler velocity variation observed in our direct view, $\sim 400 \mathrm{~km} \mathrm{~s}^{-1}$, implies values of $\sim 550 \mathrm{~km} \mathrm{~s}^{-1}$ in the plane of the orbit. The variation at FOS 4 is $\sim 250 \mathrm{~km} \mathrm{~s}^{-1}$; if we interpret this too as the projection of a velocity in the orbital plane $\left(70^{\circ}-80^{\circ}\right.$ inclination at FOS4), then true values of $\sim 750-1500 \mathrm{~km} \mathrm{~s}^{-1}$ are needed. In order to attain high enough projected flow velocities, the Soker and Behar model would therefore require FOS4 to "see" He II $\lambda 4687$ emission formed farther out in the acceleration zone than the emission in our direct view. This is not easy to arrange, because the emission process is inherently isotropic. Even more significant, one would also need to explain velocity variations that greatly exceed any credible orbital velocity. We shall comment later on possible alterations of the assumed inclination. Similar arguments apply to models in which the helium emission originates in or near the wind-wind collision region; for instance, the $\mathrm{He}_{\mathrm{I}}$ interpretation by Nielsen et al. (2007) is quite inconsistent with the FOS4 data. No matter whether one ascribes the observed Doppler variations to orbital velocities or to successive illumination of wind regions near the moving secondary star, FOS4 was expected to differ from a direct view of the star. The differences should have been far more conspicuous than our observations show.

Given the consistent time delay at FOS4, it is very difficult to imagine that the above result is mistaken; but here we can offer only conjectures to explain it. One might reconcile the data with a different inclination $i$, such that the projected orbit appears alike from both our point of view and FOS4. But that idea would "multiply the hypotheses" by requiring two unlikely assumptions: (1) the orbit plane must be tilted $20^{\circ}-25^{\circ}$ from the Homunculus midplane and (2) the azimuthal direction of the tilt must be aligned with both our line of sight and that of FOS4, so they share nearly the same projected velocities in phase with each other. In other words, both the latitude and longitude of the tilt must be suitable. This recourse seems too artificial and ad hoc to be an appealing first choice. A better explanation might be that the observed Doppler variations represent "global" changes in outward velocities, roughly spherical outflows that are not given a strong directionality by the secondary star. (An extreme form of this view might even return to the single-star mass ejection proposed by Zanella et al. 1984. We do not intend to go that far, but the question is worth contemplating.) A shock breakup model may conceivably act in a quasi-spherical way, with chaotic random velocity components during the critical time. The predominance of negative Doppler velocities may indicate that the far side of the configuration is obscured, most likely by Thomson scattering. Similar statements apply to enhanced mass loss from the primary star. Independent of these thoughts, our FOS4 results cast doubt on attempts to derive orbit parameters from apparent emission-line velocities.

\section{THE CHANGING WIND STRUCTURE DURING THE 2009 EVENT}

HST STIS data revealed that some spectral features depend on viewing direction and that the global stellar wind geometry changes during the cycle (Smith et al. 2003). The most dramatic effects occur at low latitudes, while the dense polar wind remains relatively undisturbed during an event. Departures from spherical symmetry are critical for theories of winds and instabilities in the most massive stars and we therefore reexamine selected spectral features at differing latitudes in our Gemini GMOS data. Smith et al. analyzed only three epochs after the 1998 event; 1998 March (phase $=0.04$ ), 1999 February (phase $=0.21$ ), and 2000 March (phase $=0.40)$. The improved time sampling of the GMOS observations makes it possible to monitor changes before, during, and after the 2009 event. Our results do not all agree with Smith et al., and some of the differences may signal real changes.

Hydrogen. $\mathrm{H} \alpha$ and $\mathrm{H} \beta$ emission lines are so bright in $\eta \mathrm{Car}$ that all $\mathrm{H} \alpha$ and many of the $\mathrm{H} \beta$ observations centered on the star were overexposed in Gemini GMOS observations. ${ }^{9}$ For this object $\mathrm{H} \gamma$ is usually contaminated by other emission lines, so we analyzed $\mathrm{H} \delta$. Richardson et al. (2010) have described the behavior of $\mathrm{H} \alpha$ in 2008-2009, based on a large number of observations with higher spectral resolution; but $\mathrm{H} \alpha$ samples different properties of the system because it originates at much larger radii than $\mathrm{H} \delta$ (Hillier et al. 2001).

Figure 7 exhibits variations observed in the $\mathrm{H} \delta$ P Cyg profile at several latitudes. This figure shows spectral tracings seen at the star and four locations to the SE, at several phases close to the 2009 event. GMOS observations before the event, from 2007 June to the beginning of 2009 January, revealed no significant changes in the $\mathrm{H} \delta \mathrm{P}$ Cyg profiles. At those times $(t=-353$ and -82 days), substantial P Cyg absorption was observed only at higher latitudes. This fact is usually considered evidence that the density and/or ionization structure of $\eta$ Car's current stellar wind outside an event is nonspherical (Smith et al. 2003). For most of $\eta$ Car's spectroscopic cycle, wind densities are expected

\footnotetext{
9 Gemini GMOS does not support exposure times below $1 \mathrm{~s}$.
} 


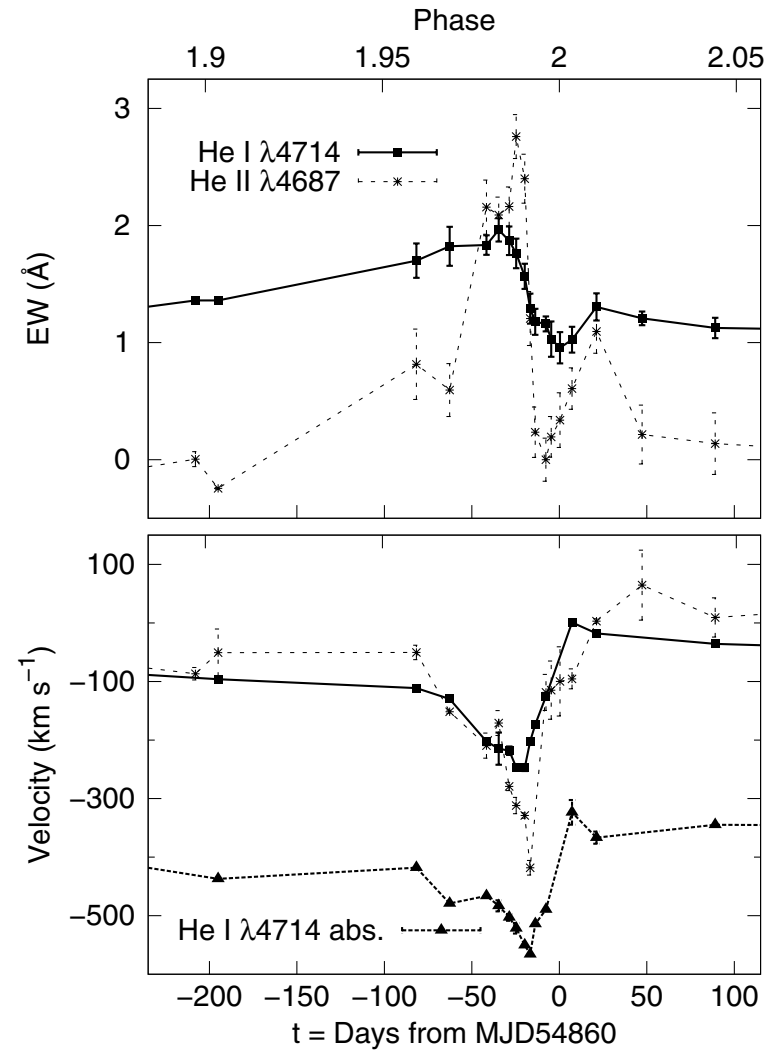

Figure 6. Equivalent width and radial velocity of the He I $\lambda 4714$ emission (filled squares) on the star during the 2009 event observed with Gemini GMOS. The radial velocity of the absorption line is also shown (filled triangles). The crosses show the values for He II $\lambda 4687$ emission; the correlation is obvious.

to be higher near the poles, in accordance with theories of equatorial gravity darkening in massive rotating stars (Maeder \& Meynet 2000; Maeder \& Desjacques 2001; Owocki 2005). But Richardson et al. (2010) have noted an alternative explanation, in which the secondary star prevents hydrogen P Cyg absorption in the primary wind. A basic obstacle to settling this question is that the fractional population of $\mathrm{H}^{0}$ in the $n=2$ level, essential for Balmer absorption, is an intricate theoretical question that has not yet been explored for $\eta$ Car's wind.

Standard theory predicts higher wind velocities at high latitudes, and Smith et al. (2003) reported such a result extending almost to $\sim-1000 \mathrm{~km} \mathrm{~s}^{-1}$ in the 2000 March STIS data (see their Figure 5). However, we find $v_{\infty} \sim 500-550 \mathrm{~km} \mathrm{~s}^{-1}$ for $\mathrm{H} \delta$ absorption at all latitudes during $\eta$ Car's normal state (Figure 7). One reason for this discrepancy may be that we use a different method to align the spectra. Smith et al. corrected for $\Delta V$ in the expanding nebula (Section 4.4 above) by aligning the blue side of $\mathrm{H} \alpha$; but that reference point in the line profile is itself affected by details of the P Cyg absorption. We use, instead, several forbidden lines that are known to originate in the Weigelt knots with constant velocities much smaller than the discrepancy in question. Also, the velocity structure of the wind may change throughout the cycle; Smith et al. used observations at phase 0.40 while our relevant observations are at phases 1.83 and 1.96. Our GMOS observation at phase 2.17 cannot be used to investigate this issue, since the $\mathrm{H} \delta$ profile had obviously not returned to its normal state. $\mathrm{H} \delta$ should be practically as good as $\mathrm{H} \alpha$ and $\mathrm{H} \beta$ for this purpose, since its absorption component is stronger, relative to emission, than in $\mathrm{H} \alpha$. A real change in the velocities and latitude structure may have occurred between 2000 and 2008.

As already discussed by Smith et al. (2003) and Stahl et al. (2005), the strong latitude dependence of Balmer P Cyg profiles does not apply during the events. In only a few days, between $t=-25$ days and -20 days, the P Cyg absorption at lower latitudes appeared and strengthened to the same depth as at higher latitudes. Strong absorption at all latitudes was observed until $t \approx+47$ days, i.e., for at least 70 days. Observations from $t=+89$ days to +344 days showed only weak absorption at low latitudes while high latitudes continued to have strong $\mathrm{P}$ Cyg profiles; the system had almost returned to its pre-event state.

P Cyg absorption is always present in the higher Balmer lines at all latitudes, consistent with their formation regions closer to the star (Weis et al. 2005). Still, during the events, their absorption deepened at lower but not higher latitudes.

Doppler velocities of Balmer lines are difficult to assess because they may include two different but unresolved parts. As noted in Mehner et al. (2011), the main velocity of $\mathrm{H} \delta$ emission remains fairly steady, while a second component appears to vary like He I (see below). The two components overlap so much that neither can be studied individually.

He I. Both the emission and the absorption components of He I vary in strength and radial velocity throughout the cycle, see Figure 6 for measurements on the star. The equivalent width of the He I emission was mostly constant during the cycle, increased before the 2009 event, and then dropped into a temporary minimum. However, the emission line never disappeared entirely as would have been expected if the event were a true eclipse. The emission lines shifted monotonically blueward throughout the cycle, terminating with an abrupt, large velocity shift of over $-100 \mathrm{~km} \mathrm{~s}^{-1}$ to velocities of about $-250 \mathrm{~km} \mathrm{~s}^{-1}$ near the event, followed by a sharp rise to almost zero radial velocity-very similar to the He II $\lambda 4687$ behavior noted in Section 4 above. The radial velocity of the absorption lines showed a similar pattern with velocity shifts between $-300 \mathrm{~km} \mathrm{~s}^{-1}$ and $-600 \mathrm{~km} \mathrm{~s}^{-1} .^{10}$

Smith et al. (2003) found that while the He I emission faded at low latitudes during the 1998 event, the emission was relatively undisturbed at higher latitudes. However, GMOS observations show that the equivalent width of the He I emission at FOS4 during the 2009 event followed basically the same patterns as the emission directly on the star.

Figure 8 shows tracings of He I $\lambda 4714$ in GMOS observations at several latitudes close to the 2009 event. Outside the event, from 2007 June to 2008 July, He I lines had strong P Cyg absorption in spectra at low latitudes and only very weak P Cyg absorption in spectra at higher latitudes (see also Smith et al. 2003). Because of their limited time sampling, Smith et al. were not able to observe changes in the He I P Cyg absorption. This led them to conclude that for most of the cycle He I absorption is present on the star but not at higher latitudes. Their Figure 18 implies that the absorption at low latitudes would disappear during the event, though this is not explicitly stated in the paper. However, in our data we find that shortly before the 2009 event, the He I absorption increased at higher latitudes. At $t=-82$ days the P Cyg absorption was strong at all latitudes. Then, over the next two months the absorption weakened at all latitudes. Between $t \approx-5$ days and $t \approx+89$ days almost no absorption was observed. By $t \approx+176$ days the He I profile had returned

\footnotetext{
10 Nielsen et al. (2007) found similar values during the 2003.5 event.
} 


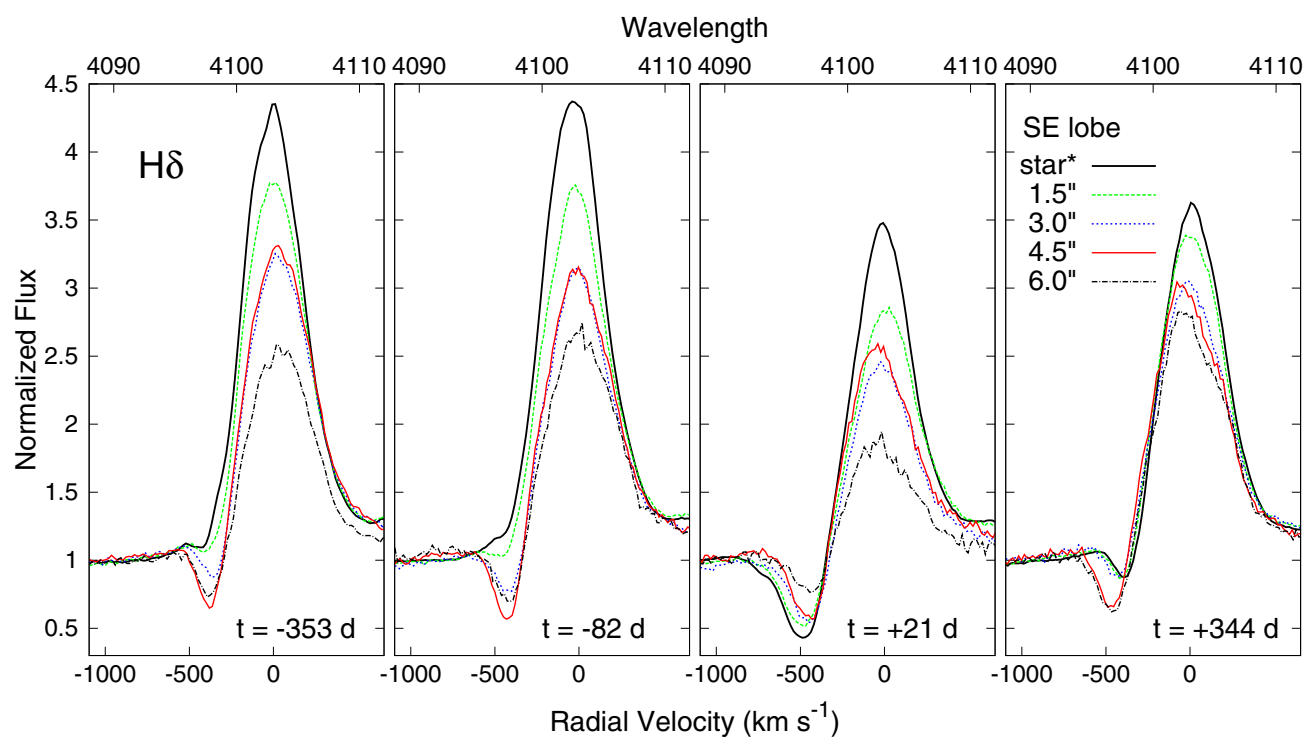

Figure 7. $\mathrm{H} \delta$ in tracings across the SE lobe in observations at $t=-353$ days (2008 February 11 , phase 1.83$), t=-82$ days (2008 November 8 , phase 1.96$), t=+21$ days (2009 February 19, phase 2.01), and $t=+344$ days (2010 January 8, phase 2.17). The key indicates the positional offset along the spectrograph slit, SE from the central source. Spectra are shifted to compensate for their $\Delta V$ "moving-mirror" redshifts. The red curve (4".5) corresponds to a spectrum which is close to position FOS4.

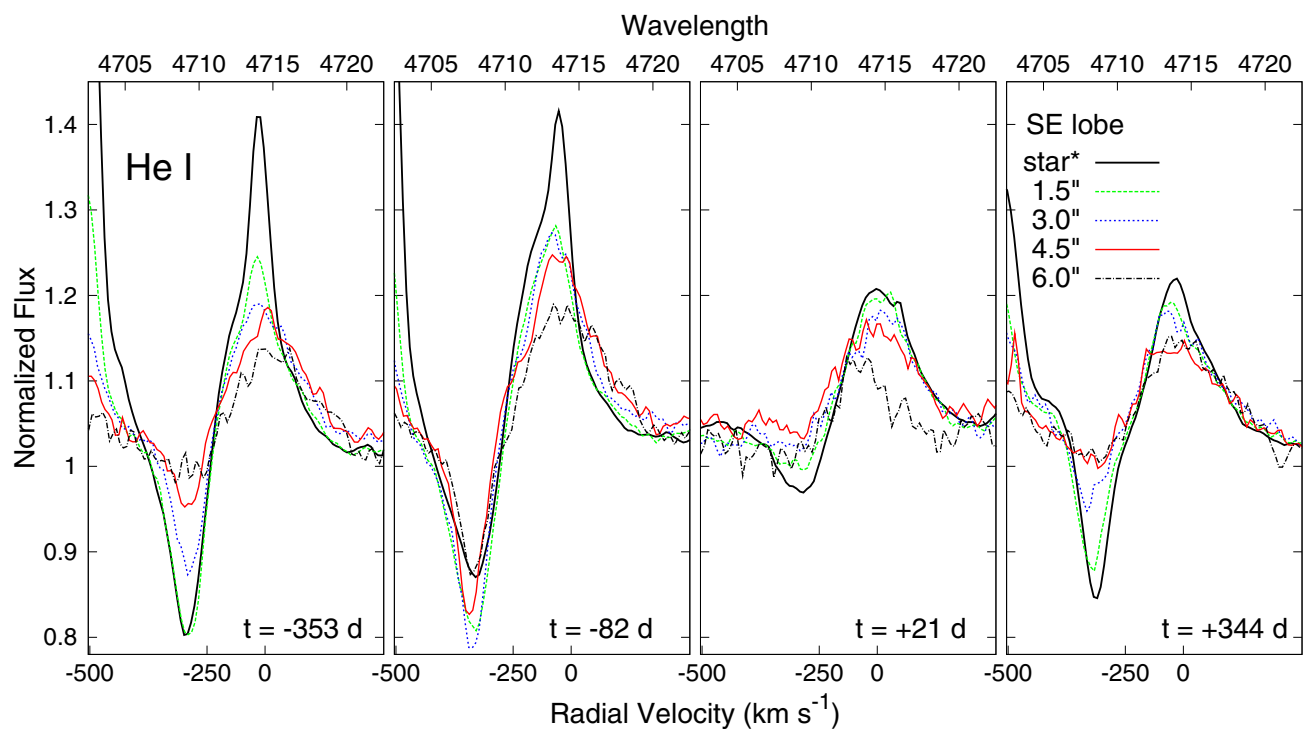

Figure 8. Tracings across the SE lobe showing He I $\lambda 4714$ emission and absorption at the same times as Figure 7. Velocities are corrected for the "moving-mirror" redshift $\Delta V$. The red curve (4".5) refers to position FOS4. Note that the large blueshift of the He I emission and absorption lines during the events, discussed in Section 5, cannot be observed in this figure since it occurs between $t \approx-70$ days and $t \approx 0$ day not sampled here. The feature at the left edge is [Fe III] $\lambda 4703$, which disappears during each spectroscopic event (Mehner et al. 2010a).

to its normal state displaying strong absorption at lower and almost no absorption at higher latitudes. Note that changes in the He I P Cyg absorption occurred at least 2 months earlier than those in the H I P Cyg absorption, and the return to the pre-event state took up to 3 months longer for He I. The presence of He I P Cyg absorption at all latitudes shortly before the event may have implications for the shell ejection model favored by Smith et al. (2003), since this observation is not directly accounted for by that model.

The geometrical volume filled by ionized helium around $\eta \mathrm{Car}$ is highly relevant, since He I absorption as well as emission lines depend on $\mathrm{He}^{+} \rightarrow \mathrm{He}^{0}$ recombination. The zones of interest are photoionized chiefly by the hot secondary star, see Humphreys et al. (2008), Mehner et al. (2010a), and references therein. If the primary wind between events has become less dense in the past few years (Mehner et al. 2010b), then the $\mathrm{He}^{+}$zone photoionized by the secondary star should now occupy a much larger volume than it did at earlier times. A crude assessment of the heliumionizing photon supply suggests that the $\mathrm{He}^{+}$zone most likely now wraps around the inner primary wind at times other than spectroscopic events. In other words, the pseudo-hyperboloidal $\mathrm{He}^{+} / \mathrm{He}^{0}$ ionization front in the primary wind may now be concave toward the primary star. If so, then most lines of sight to the primary star-including that for FOS4-must pass through some $\mathrm{He}^{+}$in the primary wind, unlike the case 10 years ago. Detailed work will be presented in a future paper.

$F e$ II. Narrow lines and broad stellar wind emission of Fe II are strong in direct spectra of $\eta \mathrm{Car}$, but much fainter in the reflected spectrum at FOS4. As already noted by Smith et al. (2003), the Fe II lines resemble Balmer lines in that the Fe II 


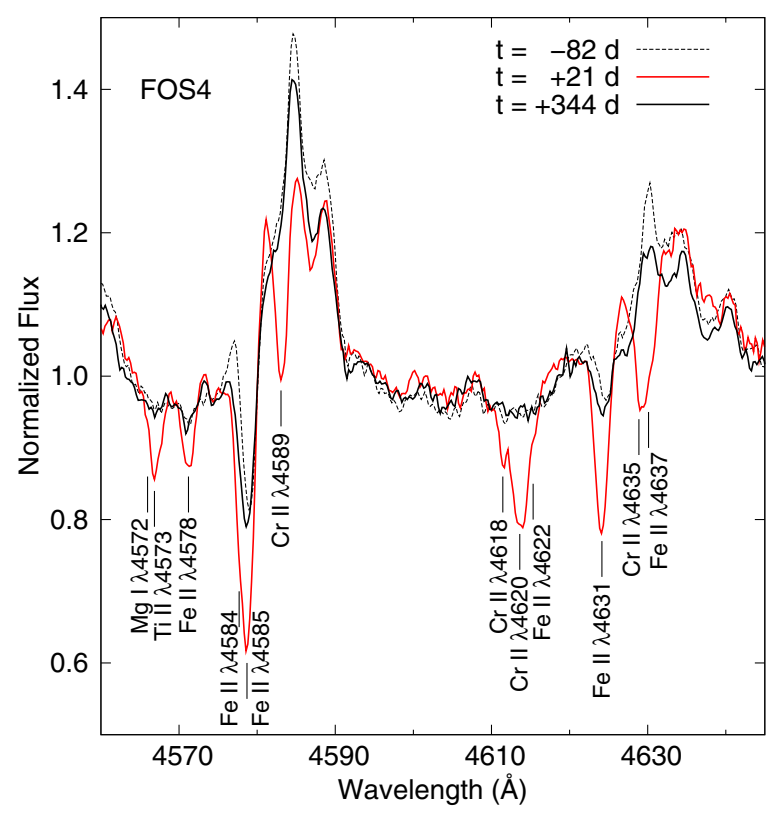

Figure 9. Absorption components at $\sim-420 \mathrm{~km} \mathrm{~s}^{-1}$ of Fe II and similar ions at FOS4 deepened for a few months during the 2009 event (solid red curve). The corresponding emission lines are weak and difficult to see in the GMOS spectra.

P Cyg absorption increases with increasing latitude and that the emission is weaker at higher latitudes. Figure 9 shows broad stellar wind emission of Fe II in spectra at FOS4. Spectra before $t \approx-82$ days showed only very weak absorption at FOS4, with maximum strength at $\sim-400$ to $450 \mathrm{~km} \mathrm{~s}^{-1}$, but the absorption feature then deepened and stayed strong until $t \approx+176$ days, i.e., for about 250 days. The absorption was strongest around $t \approx+10$ days. The deepening of Fe II absorption at FOS 4 was also observed during the 2003.5 event by Stahl et al. (2005). Other species, such as CrII, MgI, and Ti II, also developed absorption lines.

Smith et al. (2003) argued that Fe II lines are formed in the same outer wind regions as H I because these two species show similar latitude dependence and are likely to be coupled by charge exchange. However, their similarity is only true outside an event. During or close to an event the lines behave very differently; in contrast to $\mathrm{HI}$, Fe II does not develop absorption in direct view. (H I lines develop strong P Cyg absorption at lower latitudes while the pole remains almost unchanged.) Theoretically, $\mathrm{H}$ i Balmer absorption is not really like most Fe II absorption, since the hydrogen $n=2$ level has a much higher energy than most Fe II lower levels $(10 \mathrm{eV}$ compared to $0-3 \mathrm{eV}$ ). The observed Balmer absorption lines may involve $\mathrm{H}^{+} \rightarrow \mathrm{H}^{0}$ recombination, analogous to $\mathrm{He}$ I noted above.

$N$ II. Unlike any other known low-ionization features, the $\mathrm{N}$ II multiplet at $\lambda \lambda 5668-5712$ varied in Doppler velocity like the He I lines and did not have steady components. Our observations of these lines have been reported separately (Mehner et al. 2011); evidently they originate in fairly normal zones of the primary wind but are excited by UV photons from the secondary star. Their net Doppler velocities seen at position FOS4 resembled those in the direct view, again presenting the same puzzle seen in helium lines regarding orbital motion. Unfortunately, we cannot say much more about them concerning the 2009 event, because they require high spatial resolution (i.e., HST) in order to eliminate strong contamination by un- related emission lines that represent ejecta outside the stellar wind.

\section{CRITICAL DIFFERENCES IN THE 2009 EVENT-A SUMMARY, CONCLUSIONS, AND PROBLEMS}

\subsection{Primary Results}

We monitored $\eta$ Car with HST WFPC2 and Gemini GMOS throughout its 2009 spectroscopic event. Good time coverage with the GMOS slit oriented at a constant position angle made it possible to monitor spectroscopic changes as seen from a range of directions, some of them via reflected light. In this paper, we have described several important differences compared to previous events, some of them quite unexpected. These results lend strong support to one important concept, the idea of shock breakup and collapse near periastron. Regarding other aspects of the problem, the new information helps to exclude some proposed models, while - as usual for this topic — it also deepens some of the puzzles.

HST WFPC2 observations show that the minimum in the UV was much deeper for the 2009 event than for the 2003.5 event (Section 3). One possible explanation involves a massloss outburst or similar phenomenon, discussed in Section 6.2 below.

Contrary to expectations, we find that the behavior of the He II equivalent width and radial velocity reflected at polar location FOS4 are very similar to direct observations of the star. For this purpose an observed light-travel-time delay $\Delta t \approx 18$ days and moving-mirror redshift $\Delta V \approx+100 \mathrm{~km} \mathrm{~s}^{-1}$ must be taken into account. Since the observed $\Delta t$ agrees well with the predicted value, it confirms that FOS4 really does "see" the star from a polar direction. The observed radial velocity behavior at FOS4 is a surprise, because, contrary to proposed models, it fails to show major differences compared to our direct view of the star. This statement includes other effects as well as the orbital velocity.

We found that the "second He II $\lambda 4687$ episode" in 2009 was strongly anti-correlated with the X-rays, like a time reversal of the main $\lambda 4687$ outburst seen in 2003 and 2009. In Section 4.3 , we argued that this result strongly supports the shock structure breakup/disintegration/collapse hypothesis, first proposed a decade ago as an explanation for X-ray behavior during a spectroscopic event. Strictly speaking this idea has not been proven, but now the following facts together have made it the most probable model.

1. Likely physical conditions near periastron appear suitable for the relevant instabilities (Soker 2003; Martin et al. 2006a; Soker \& Behar 2006; Parkin et al. 2009; Akashi et al. 2011).

2. The He II $\lambda 4687$ outburst was not yet recognized when shock breakup was first proposed in 2001-2003, see Section 4.3 above. But after it was discovered and measured, this phenomenon turned out to be very well suited to the idea (Martin et al. 2006a).

3 . The 2009 event featured a new development, the second $\lambda 4687$ episode about 30 days after the first. As we discussed in Section 4.3, this was beautifully accordant with the earlier-than-expected reappearance of 2-10 keV X-rays.

4. The timescales are reasonable as noted earlier.

5. From a theoretical viewpoint, other proposed explanations (especially eclipses, which some authors favored until two or three years ago) have much worse difficulties (Martin et al. 2006a; Parkin et al. 2009). 
Shock breakup is still a hypothesis, albeit a very strong one, not a quantitative theory. Soft X-ray production during the collapse, and structural recovery later, has scarcely been investigated yet. A broad range of parameter space must be explored.

Let us emphasize that shock breakup would explain only some aspects of a spectroscopic event. Other phenomena, more fundamental for the basic physics of $\eta$ Car, are probably required in order to explain the spectroscopic and photometric changes. Some of them are noted in Section 6.2 below.

Results on spectral features of hydrogen, He I, and other species (Section 5) are too diverse to summarize briefly. Hydrogen P Cyg profiles at different latitudes throughout the cycle behave as already discussed in Smith et al. (2003). Outside the 2009 event, H I P Cyg absorption is observed at higher latitudes but not at lower, while during the event H I P Cyg absorption is also strong at lower latitudes. GMOS data show that the P Cyg absorption at lower latitudes appeared suddenly within only a few days and was present for at least 70 days. We do not find a higher terminal velocity at higher latitudes as found by Smith et al. (2003). The exact reasons have to be addressed in the future.

Helium P Cyg profiles showed an additional incident not discussed by Smith et al. Those authors found that outside the events He I P Cyg absorption is present at low latitudes but absent at higher latitudes, while during the events the absorption disappears at low latitudes, too. Our GMOS data show that shortly before the 2009 event He I absorption increased at higher latitudes to similar strength as at low latitudes. Then the absorption decreased slowly at all latitudes. Changes in the He I lines were observed already two months before the $\mathrm{H}_{\mathrm{I}}$ lines showed any changes and they returned to their normal "no-event" state up to three months later than the H I lines.

Fe II absorption, only present at higher latitudes, became very strong during the 2009 event for several months and behaved differently than the H I lines. The special N II lines near $5700 \AA$ with similar radial velocity variations as the $\mathrm{He}$ I lines give different information and will become unique diagnostics in the future if enough data are obtained both on the star and at position FOS4.

Further analysis is required with regard to the cause of those observed latitude-dependent changes throughout the events. Is a minor shell ejection sufficient to explain them? Or do changing ionization fronts in the primary wind caused by influences of the secondary star and/or the moving wind-wind shock structure play a role?

\subsection{Unsolved Problems}

A number of essential questions have not yet been answered even after years of observation and discussion. Each requires theoretical work that no one has attempted at a realistic level of detail, and our 2007-2010 results are pertinent to some of them.

Does the secondary star accrete material during a spectroscopic event? This possibility has been emphasized especially by Soker and colleagues (e.g., Soker 2003, 2007; Soker \& Behar 2006; Kashi \& Soker 2009a). As we noted in Section 4.3, near periastron the primary wind may entirely suppress the secondary wind, thereby allowing accretion onto the secondary star. Some of the consequences can be separated from other aspects of those authors' model. In our view, accretion may explain a long-standing paradox concerning $\eta$ Car's He I emission lines (Davidson 1999; Humphreys et al. 2008). These features depend chiefly on photoionization by the hot secondary star, they weaken during a spectroscopic event, and they were not present before the 1940s. Merely saying that "the secondary star was engulfed in dense gas" does not constitute an explanation, since helium-ionizing photons $(h v>24.6 \mathrm{eV})$ inevitably generate He I recombination lines in the primary wind even at densities far above normal. Roughly speaking, the brightness of He I emission measures the far-UV photon supply. ${ }^{11}$ However, as Soker (2007) noted, accretion can lower the secondary star's effective temperature by slightly expanding its photosphere. Even a modest reduction in temperature substantially cuts the output of helium-ionizing photons (Mehner et al. 2010a, and references therein). Humphreys et al. remarked that $\eta$ Car's mass-loss rate may have considerably exceeded $10^{-3} M_{\odot} \mathrm{yr}^{-1}$ in the years 1900-1940; the primary star's wind density near the secondary star then may usually have been as large as it is today near periastron-i.e., perhaps enough to crush the secondary wind. (See also Kashi \& Soker 2009b, concerning $\eta$ Car's Great Eruption.)

Accretion thus provides a satisfying explanation to the He I puzzle; but is it correct? Helium emission lines did not disappear in 2009 (Figure 6), but on the other hand this was presumably the least dense spectroscopic event on record. Akashi et al. (2011) have recently presented three-dimensional gas dynamical numerical simulations, which indicate that the secondary star should indeed accrete mass from the dense primary wind close to periastron passage, but this is an extraordinarily complex nonspherical problem, depending on many insecure parameters; therefore, at present it is reasonable to view accretion as likely and theoretically desirable, but not yet proven. If the primary wind continues its secular decline in density, accretion near periastron should decrease and may eventually cease to occur.

Does each spectroscopic event include a mass-loss outburst by the primary star? This suggestion arose long ago because eclipse models appeared inadequate (Davidson 1999), and because Zanella et al. (1984) had discussed the same idea in a single-star context. Today the shock breakup concept probably explains the X-ray and He II $\lambda 4687$ behavior, but other spectral features involve larger spatial regions in the unshocked primary wind. Throughout this paper, we have mentioned indications that the primary wind may have been disturbed. Most of them have been noted before, and none is entirely satisfying, but their combination is highly suggestive.

1. Our UV photometry showed much deeper minima in 2009 than in 2003. In Section 3, we noted why this may be evidence for extra material in the system during the early stages of the event. Qualitatively, a higher density due to temporary causes would affect the Fe II forest and UV photometry in the desired direction. Shock breakup, by contrast, does not provide such an obvious explanation. A similar argument can be made regarding near-infrared free-free emission.

2. Temporarily enhanced gas densities would help to destabilize the shock structure, while also supplying extra energy for He II $\lambda 4687$, as Martin et al. (2006a) explained.

3. In Section 4.3, we noted that the 2008-2009 X-ray crash "should have" occurred later than predicted, since it is now

11 Mehner et al. (2010a) estimated $T_{\text {eff }} \approx 40,000 \mathrm{~K}$ and $10^{5} L_{\odot} \lesssim L \lesssim 10^{6} L_{\odot}$ for $\eta$ Car's secondary star. Such an object produces $10^{47.7}$ to $10^{49.0}$ helium-ionizing photons per second, and we estimate that the logarithmic upper half of this range is sufficient to account for the observed equivalent widths of $\eta$ Car's He I lines. In addition to the reasoning used for nebulae (Osterbrock \& Ferland 2006), one must include a special enhancement factor explained in Section 6 of Humphreys et al. (2008). 
known that the primary wind decreased after 2003 (Mehner et al. 2010b; Kashi \& Soker 2009a; Corcoran et al. 2010). Extra ejected material dependent on other factors, however, would have made the secular density trend temporarily irrelevant.

4. In Section 4.4, we stated that a reflected polar view of the 2009 event closely resembled the behavior seen directly, including the Doppler variations. Any effect involving orbital motion should have appeared different between the viewpoints. A conceivable explanation is that velocities during the event may have represented instead a varying quasi-spherical outward flow from the primary star.

5. Complicated changes in P Cyg absorption features, described in Section 5 above, suggest that column densities increase especially at low latitudes during an event (compare Smith et al. 2003).

6. If the Eddington limit is taken into account (and, perhaps, rotation), tidal forces are very likely strong enough near periastron to alter the primary-wind-acceleration zone (Davidson 1997). Various instabilities may have broadened the flow.

Of course, we do not claim that a mass ejection event would explain everything, but it would help with all the above points, and there is no clear evidence against it.

The outburst conjecture has both milder and stronger variants. For instance, the total mass-loss rate might remain constant during the event, but its latitude dependence briefly changes, causing densities to increase at low latitudes (Smith et al. 2003). At the other extreme, the base of the wind might be affected, not just its acceleration zone. That case would signal an undiagnosed surface instability in the star itself. How can this question be investigated? As a beginning, one might explore theoretically whether the photometric and spectroscopic observations can be explained by shock breakup without a primary-wind outburst or disturbance.

The primary wind density has been decreasing and may continue to do so until the star has a normal line-driven wind (Mehner et al. 2010b). If so, then the wind-wind shocks will very likely become stable even at periastron, ending the series of X-ray and He II "events" as we know them today. This might even occur within the next two or three 5.5 year cycles. If, on the other hand, the secondary star is capable of triggering a tidal/radiative outburst near periastron as discussed above, then the X-ray events will continue to occur. In that case the X-ray luminosity will be diminished, but not as severely as the wind density. (This statement is based on simple considerations of the wind-wind momentum balance and cooling rate.)

How can the orbital parameters be estimated? The FOS4 results bolster our opinion that observed Doppler velocities cannot be used for this purpose until they are much better understood. There have been attempts to derive orbit parameters by directly identifying $\eta$ Car's observed Doppler variations with orbital velocities, like a classical spectroscopic binary (e.g., Damineli et al. 1997; Nielsen et al. 2007; Kashi \& Soker 2008). This approach is questionable because neither the emitting nor the absorbing gas is expected to share the motion of either star, efforts of this type have contradicted each other, and now our FOS4 results create even greater doubts. The observed velocities are more likely to represent samples of wind regions near the moving secondary star, but in this case one needs to model the complicated varying threedimensional spatial volumes, local absorption may vary, and the FOS4 puzzle still applies. Thus, until more is known about the projected velocities, orbit parameters must be based on other considerations.

The semimajor axis $a$ is uncontroversial, 16-19 AU for a total mass in the likely range $130-220 M_{\odot}$. Rough limits on the orbital eccentricity $\epsilon$ are usually based on the duration of a spectroscopic event. Most likely the main parts of an event occur at orbital longitudes within $\pm 90^{\circ}$ of periastron, i.e., while the two stars are separated less than twice the periastron distance. Since the time required to move from $-90^{\circ}$ to $+90^{\circ}$ is $\Delta t_{180} \approx(1200$ days $) \times(1-\epsilon)^{3 / 2}$, eccentricities between 0.80 and 0.93 seem reasonably consistent with Figure 4. Most likely $0.84<\epsilon<0.91, \Delta t_{180} \sim 30-80$ days, and the periastron separation is between 1.5 and 2.8 AU. If the first and second $\lambda 4687$ episodes in 2009 occurred before and after periastron, the most likely time of periastron was close to $t \approx 0$.

The orbit orientation is more difficult to guess. Very probably the orbit plane is close to the Homunculus midplane, with inclination $i \approx 45^{\circ}$ (Davidson et al. 2001); any substantially different inclination seems unlikely in view of tidal friction near periastron and would be outside the scope of our discussion here. But what orientation does the orbit have within that plane? Most authors quote $\omega$, the longitude of periastron as defined in textbooks. For $\eta$ Car, $\omega=270^{\circ}$ would indicate that the secondary star is on the far side of the primary at periastron, while $\omega=180^{\circ}$ represents an orbit whose major axis is perpendicular to our line of sight. Okazaki et al. (2008), Parkin et al. (2009), and Groh et al. (2010) all favored $\omega \approx 240^{\circ}$ based on X-rays and other data, but these are not strictly independent estimates, since they shared a number of insecure assumptions. One symptom of uncertainty is that Ishibashi (2001) found $\omega \approx 200^{\circ}$ by similar reasoning. (The difference between $200^{\circ}$ and $240^{\circ}$ is substantial, because the latter value places the secondary star almost behind the primary at periastron, while $200^{\circ}$ gives a more "sideways" view.) Ishibashi's analysis was far simpler, but the other, more elaborate analyses required more assumptions. Moreover, Kashi \& Soker (2009b) used the $\mathrm{X}$-rays to find an orientation much different from those cited above. Many neglected effects can have major consequences on the wind simulations. For example, variable inhomogeneities commonly exist in stellar winds, may be especially crucial for $\eta$ Car's X-ray luminosity, and are extremely difficult to model. Therefore $\omega$ is in fact quite uncertain.

Another datum may help to indicate $\omega$. Mehner et al. (2010a) found conspicuous brightness maxima in the high-excitation [Ne III] and [Fe III] emission along our line of sight to the star, around phase 0.95 or $t \sim-110$ days in 2003 - i.e., considerably before the event had begun. What is special about that part of the orbit? Considering the nature of those emission lines (see Mehner et al.), it probably signaled the time when UV photons from the secondary star can pass along our line of sight entirely within the rarified secondary wind, i.e., within the pseudo-hyperboloidal "shock cone." Apart from a geometrical correction noted below, this corresponds to the time when the secondary star passed through the projection of our line of sight onto the orbit plane, indicating a value for $\omega$. A few calculations yield the result

$$
\omega \approx 259^{\circ}-(1-\epsilon) \times 201^{\circ},
$$

where $\epsilon$ is the orbital eccentricity. Here we have included corrections of $5^{\circ}-9^{\circ}$ for the fact that the shock structure is not exactly coaxial with the line between the two stars, a consequence of orbital motion across the primary-wind flow. The quasi-formal error in this result is only a few degrees, 
because the angular motion is slow at the relevant time, but the chief uncertainty, of course, concerns the proposed interpretation of the observed effect. ${ }^{12}$

Did an eclipse play any role in the spectroscopic event? With the orbit orientation advocated by Kashi \& Soker (2009b), the answer is "almost none" because the secondary star and shocks do not pass behind the primary wind near the time of an event. Most other proposed models, however, employ different orientations which may allow the primary wind to obscure the secondary star and interaction region either near periastron or in the following weeks. Figure 10 shows a projected view for one plausible set of orbital parameters. Suppose, for example, that these are correct and that the opaque radius in the primary wind is 2-3 AU (Hillier et al. 2001) as seemed likely before the recent decrease in the wind density. In that case, the active region should be at least partially obscured for roughly 30 days around periastron. Martin et al. (2006a) remarked that an eclipse of this type would not dominate the observational record, because the main stages of the spectroscopic event occur earlier or later. In 2009, however, He II $\lambda 4687$ showed interesting nonzero behavior during the time when an eclipse is suspected (Figures 3 and 4). Conceivably, this feature may have been heavily obscured near periastron in 2003 , but was more visible in 2009 because the wind density had decreased; if true, this might affect the reasoning in Section 4.2 above. On the other hand, the onset of a quasi-eclipse should be gradual, because the relevant zones of the primary wind are quite diffuse and the $\lambda 4687$ emission region is most likely somewhat extended. Thus it would be difficult to explain the abrupt $\lambda 4687$ flux decrease in terms of an eclipse. This question obviously needs more investigation.

In summary, available data are not quite adequate for the above problems but the scarcity of theoretical work is far worse. One reason for this deficiency is obvious: too much is known about $\eta$ Car. Spherical and even axial symmetry are doubtful approximations, while non-routine atomic processes and radiative transfer play substantial roles. Similar statements may apply to other very massive stars but few details are known for them. If $\eta$ Car cannot be explained quantitatively with existing theory, then existing theory should not be considered realistic. In other words, the goal of solving these problems is not merely a challenge; it can be regarded as a valid test of contemporary astrophysics.

We thank the staff and observers of the Gemini-South Observatory in La Serena for their help in preparing and conducting the observations, and Beth Perriello at STScI for assistance with HST observing plans.

\section{APPENDIX}

\section{CONCERNING "PHASE” AND THE 5.5 YEAR PERIOD}

We know from experience that varying definitions of "phase" in $\eta$ Car's 5.5 year cycle have caused confusion. There have been at least three difficulties, some of them matters of principle.

1. Most authors specified their zero points $t_{0}$ to coincide with various observed phenomena, e.g., the disappearance of some spectral feature, but the chosen phenomena are of

12 Incidentally, the high-excitation precursor peak should recur in 2014 April-May, and, if our interpretation is valid, it should be broader and less conspicuous than in 2003. Reason: the decreased primary wind should have broadened the opening angle of the wind-wind "shock cone."

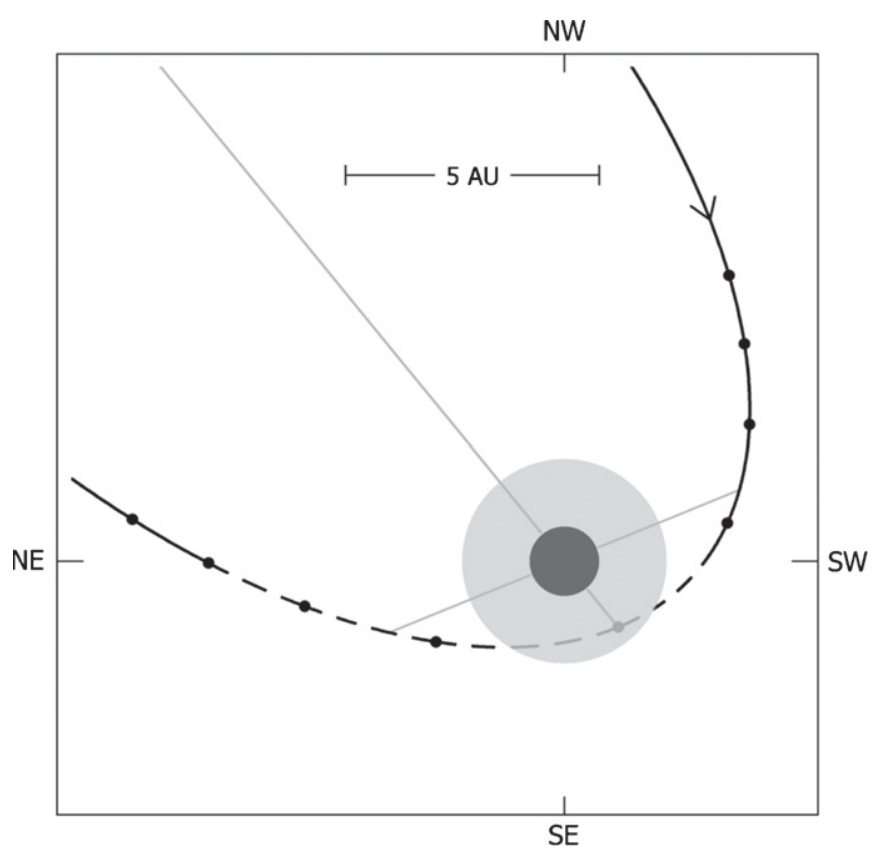

Figure 10. Projected apparent view of the companion star's motion for an orbit with $\epsilon=0.875, \omega=240^{\circ}$, and $i=45^{\circ}$. The dot marks show positions at 20 day intervals; at periastron the secondary star is on the far side of the primary. The large shaded circle has $r=2 \mathrm{AU}$. Two straight lines show the major axis and latus rectum, and the nodes are located along the horizontal line NE-SW. Labels NW, SW, SE, and NE indicate approximate directions in the sky. Figure 4 in Mehner et al. (2011) shows approximately the same orbit as seen from a different direction. Caveat: $\eta$ Car's true orbital parameters are quite uncertain; see the text.

doubtful significance, because spectroscopic events are too poorly understood to identify any observable quantity with a basic physical or geometric parameter in the binary system.

2. This style of reckoning gives a misleading impression of high precision and future repeatability. In fact, successive events are known to differ from each other. Given the continuing changes in $\eta$ Car (see references in Section 1), any observable quantity may shift its phase in the cycle. Terms such as "ephemeris" can be very misleading in circumstances like these.

3. The adopted periods and zero points have varied from paper to paper. We have identified at least seven different phase definitions of this type in the literature, with periods spanning a range of 10 days and zero points ranging across 20 days. Authors have repeatedly changed their definitions.

Fortunately, one simple definition has remained constant and is greatly preferable in terms of logic and procedure. For the $\eta$ Car HST Treasury Program Archive (http://etacar.umn.edu/), "phase" was intentionally defined in a calendar-based rather than a phenomenon-based way. This choice was made specifically to avoid the pitfalls listed above. Its period and zero point are 2023.0 days and MJD 50814.0 = J1998.000 exactly. Phase 2.000 occurred at MJD 50860.0 = J2009.077. The integer quantities help to minimize calculative errors, and, more important, they discourage any impression that $t=0$ represents some critical time with respect to physics. (Periastron is thought to occur fairly close to $t=0$, but this categorically plays no role in the definition.)

This system has been in use since 2003 without alteration; its adopted period of 2023.0 days continues to agree with the best measurements within less than $1 \sigma$ (Damineli et al. 2008b; Fernández-Lajús et al. 2010); and it is used in the Treasury 
Table 4

Photometric $t_{\text {phot }}$ MJD's for $\eta$ Car's 1998.0, 2003.5, and 2009.0 Spectroscopic Events

\begin{tabular}{lcccc}
\hline \hline Waveband & 1998.0 & 2003.5 & 2009.0 & \multicolumn{2}{c}{ Difference } \\
& $t_{\text {phot }}$ & $t_{\text {phot }}$ & $t_{\text {phot }}$ & $\Delta t_{\text {phot }}$ \\
\hline$K^{\mathrm{a}}$ & $50802.6 \pm 1.8$ & $52824.7 \pm 0.5$ & $\ldots$ & $2022.1 \pm 1.9$ days \\
$H^{\mathrm{a}}$ & $50803.3 \pm 1.3$ & $52825.9 \pm 0.4$ & $\ldots$ & $2022.6 \pm 1.4$ days \\
$J^{\mathrm{a}}$ & $50807.0 \pm 1.5$ & $52826.8 \pm 0.4$ & $\ldots$ & $2019.8 \pm 1.6$ days \\
$330 \mathrm{~nm}$ & $\ldots$ & $52827.8 \pm 1.7$ & $54850.0 \pm 1.7$ & $2022.2 \pm 2.4$ days \\
$250 \mathrm{~nm}$ & $\ldots$ & $52826.1 \pm 1.1$ & $54842.7 \pm 0.6$ & $2016.6 \pm 1.3$ days \\
\hline
\end{tabular}

Note. ${ }^{\text {a }}$ Feast et al. (2001) and Whitelock et al. (2004).

Program archive, which is the largest easily accessible source of data on $\eta$ Car (reduced data of all HST STIS, VLT UVES, and Gemini GMOS observations are available). Hence there is no reason to substitute any later, arbitrary system. In order to minimize present and future confusion, and for the other reasons noted above, it is the obvious standard.

Timing measurements are valuable because they may indicate changes between successive events. One particular operational procedure achieves the best precision and reproducibility, as follows. Various observable quantities-e.g., photometry at most wavelengths - attain brief maxima $Q_{\max }$ at a particular stage in a spectroscopic event, and then briskly fall to their local minima $Q_{\min }$ (or vice versa). The times of maximum and minimum are imprecise, but for some observables the time of midpoint, when $Q$ is the average of maximum and minimum, is more precise than anything else in the spectroscopic event. This is true mainly because the descent is very rapid at that time. If $Q(t)$ is fairly smooth and if enough data points are available, then the most robust measurement protocol is as follows.

1. Estimate the value of $Q_{\max }$ but ignore its time $t\left(Q_{\max }\right)$ which may be ill-defined. A local quadratic fit may be appropriate, but fortunately there is no need for very high precision in $Q_{\max }$ (see below).

2. Do the same for $Q_{\min }$ and calculate the midpoint $Q_{\mathrm{m}}=$ $\left(Q_{\min }+Q_{\max }\right) / 2$.

3. Then estimate the midpoint time $t_{\mathrm{m}}=t\left(Q_{\mathrm{m}}\right)$, based only on the data points that are nearest to it. If many data points are available, for instance, one might use only those in the range $Q_{\mathrm{m}} \pm \Delta Q$, where $\Delta Q \approx\left(Q_{\max }-Q_{\min }\right) / 4$. In favorable circumstances either a linear or a cubic fit gives practically the same result.

In effect this is a specialized form of interpolation. Each step employs only a local subset of the data, so local irregularities in behavior around the maximum and minimum do not affect $t_{\mathrm{m}}$ much. In the case of $\eta$ Car's events, $t_{\mathrm{m}}$ is only weakly sensitive to the estimated values $Q_{\max }$ and $Q_{\min }$. This is true because, for most observables, the curve $Q(t)$ is approximately antisymmetric near the midpoint and the rate of descent is quite rapid there. Note that theoretical significance plays no role in the empirical reasoning. For an especially successful example, $\eta$ Car's " $J$ " magnitudes in 2003 reported by Whitelock et al. (2004), the rms formal error in $t_{\mathrm{m}}$ is only \pm 0.4 day. We employed the same method for some details in Section 4.1 above.

Fernández-Lajús et al. (2010) used a method that is more general but is not as well suited to $\eta$ Car in particular. In effect, their procedure mixes data in a broader time interval, thereby allowing a stronger dependence on behavior details around $Q_{\max }$ and $Q_{\text {min }}$. Damineli et al. (2008b) defined a phase zero point based on an extrapolation, which of course is inherently far less robust than interpolation.
Fernández-Lajús et al. (2009, 2010) report high-quality ground-based photometry since 2003, but it is extremely unfortunate that the earlier $J H K$ program described by Feast et al. (2001) and Whitelock et al. (2004) was forced to close before the 2009.0 event. Their results give precise estimates of a time related to the 2003.5 event, and their earlier data give fairly good results for the 1998.0 event (see below). Here we estimate the 2003-2009 inter-event time interval from our HST UV photometry (Section 3), because these data represent the stellar wind with little contamination by ejecta at $r \gtrsim 0$.'15. Unfortunately, the temporal sampling is sparse compared to ground-based photometry.

Table 4 lists the results for $t_{\mathrm{m}}$ in three events and five wavebands. Here we use $J H K$ photometry by Feast et al. and Whitelock et al. cited above, and our UV photometry. JHK wavelengths are pertinent because light reflected by dust in the ejecta is less than for $U B V R I$. The quoted uncertainties are semi-formal rms standard errors based on observed scatter or deviation of the data points and other details; these appear to be realistic, judging from the scatter in the three periods based independently on $J, H$, and $K$. Note that $t_{\mathrm{m}}$ for a particular event may depend on wavelength; it is the difference between events that we are concerned with here. The JHK data indicate an interval $2021.5 \pm 0.9$ days between the 1998.0 and 2003.5 events; this compares with $2022.7 \pm 1.3$ days found by Damineli et al. (2008b) with less suitable methods and a much larger set of data, and $2022.8 \pm 0.5$ days found by Fernández-Lajús et al. (2010) from ground-based photometry in 2003-2009. The HST $330 \mathrm{~nm}$ value is obviously consistent with these, but the $250 \mathrm{~nm}$ result appears discordant. This may be a real effect; the $250 \mathrm{~nm}$ wavelength region physically differs from the others because it represents the Fe II forest (Section 3). Unfortunately, there is no assurance that UV photometry will be possible during the next, 2014.6 event.

\section{REFERENCES}

Akashi, M., Kashi, A., \& Soker, N. 2011, arXiv:1106.2438v1

Altamore, A., Baratta, G. B., Cassatella, A., Rossi, L., \& Viotti, R. 1986, in New Insights in Astrophysics, Eight Years of UV Astronomy with IUE, ed. E. J. Rolfe (ESA SP-263; Noordwijk: ESA), 303

Cassatella, A., Giangrande, A., \& Viotti, R. 1979, A\&A, 71, L9

Cleveland, W. S. 1979, J. Am. Stat. Assoc., 74, 829

Cleveland, W. S., \& Devlin, S. J. 1988, J. Am. Stat. Assoc., 83, 596 Corcoran, M. F., Hamaguchi, K., Pittard, J. M., et al. 2010, ApJ, 725, 1528

Corcoran, M. F., Ishibashi, K., Davidson, K., et al. 1997, Nature, 390, 587 Damineli, A. 1996, ApJ, 460, L49

Damineli, A., Conti, P. S., \& Lopes, D. F. 1997, New Astron., 2, 107

Damineli, A., Hillier, D. J., Corcoran, M. F., et al. 2008a, MNRAS, 386, 2330

Damineli, A., Hillier, D. J., Corcoran, M. F., et al. 2008b, MNRAS, 384, 1649

Damineli, A., Kaufer, A., Wolf, B., et al. 2000, ApJ, 528, L101 
Damineli, A., Stahl, O., Wolf, B., Kaufer, A., \& Jablonski, F. J. 1999, in ASP Conf. Ser. 179, Eta Carinae at The Millennium, ed. J. A. Morse, R. M. Humphreys, \& A. Damineli (San Francisco, CA: ASP), 221

Davidson, K. 1997, New Astron., 2, 387

Davidson, K. 1999, in ASP Conf. Ser. 179, Eta Carinae at The Millennium, ed. J. A. Morse, R. M. Humphreys, \& A. Damineli (San Francisco, CA: ASP), 304

Davidson, K. 2002, in ASP Conf. Ser. 262, The High Energy Universe at Sharp Focus: Chandra Science, ed. E. M. Schlegel \& S. D. Vrtilek (San Francisco, CA: ASP), 267

Davidson, K. 2005, in ASP Conf. Ser. 332, The Fate of the Most Massive Stars, ed. R. Humphreys \& K. Stanek (San Francisco, CA: ASP), 101

Davidson, K., Ebbets, D., Weigelt, G., et al. 1995, AJ, 109, 1784

Davidson, K., Gull, T. R., Humphreys, R. M., et al. 1999a, AJ, 118, 1777

Davidson, K., Ishibashi, K., Gull, T. R., \& Humphreys, R. M. 1999b, in ASP Conf. Ser. 179, Eta Carinae at The Millennium, ed. J. A. Morse, R. M. Humphreys, \& A. Damineli (San Francisco, CA: ASP), 227

Davidson, K., Ishibashi, K., Gull, T. R., Humphreys, R. M., \& Smith, N. 2000, ApJ, 530, L107

Davidson, K., Martin, J., Humphreys, R. M., et al. 2005, AJ, 129, 900

Davidson, K., Smith, N., Gull, T. R., Ishibashi, K., \& Hillier, D. J. 2001, AJ, 121,1569

Feast, M., Whitelock, P., \& Marang, F. 2001, MNRAS, 322, 741

Fernández-Lajús, E., Fariña, C., Calderón, J. P., et al. 2010, New Astron., 15, 108

Fernández-Lajús, E., Fariña, C., Torres, A. F., et al. 2009, A\&A, 493, 1093

Fernández-Lajús, E., Gamen, R., Schwartz, M., et al. 2003, IBVS, 5477, 1

Gaviola, E. 1953, ApJ, 118, 234

Groh, J. H., Nielsen, K. E., Damineli, A., et al. 2010, A\&A, 517, A9

Gull, T. R., Davidson, K., \& Ishibashi, K. 2000, in AIP Conf. Proc. 522, Cosmic Explosions: Tenth Astrophysics Conference, ed. S. S. Holt \& W. W. Zhang (Melville, NY: AIP), 439

Hillier, D. J., Davidson, K., Ishibashi, K., \& Gull, T. 2001, ApJ, 553, 837

Humphreys, R. M., Davidson, K., \& Koppelman, M. 2008, AJ, 135, 1249

Humphreys, R. M., et al. 1999, in ASP Conf. Ser. 179, Eta Carinae at The Millennium, ed. J. A. Morse, R. M. Humphreys, \& A. Damineli (San Francisco, CA: ASP), 107

Ishibashi, K. 2001, in ASP Conf. Ser. 242, Eta Carinae and Other Mysterious Stars: The Hidden Opportunities of Emission Spectroscopy, ed. T. R. Gull, S. Johannson, \& K. Davidson (San Francisco, CA: ASP), 53

Ishibashi, K., Corcoran, M. F., Davidson, K., et al. 1999a, ApJ, 524, 983

Ishibashi, K., Davidson, M. F., Corcoran, K., et al. 1999b, in ASP Conf. Ser. 179, Eta Carinae at The Millennium, ed. J. A. Morse, R. M. Humphreys, \& A. Damineli (San Francisco, CA: ASP), 266

Kashi, A., \& Soker, N. 2008, MNRAS, 390, 1751

Kashi, A., \& Soker, N. 2009a, ApJ, 701, L59

Kashi, A., \& Soker, N. 2009b, MNRAS, 397, 1426

Maeder, A., \& Desjacques, V. 2001, A\&A, 372, L9

Maeder, A., \& Meynet, G. 2000, A\&A, 361, 159

Martin, J. C., Davidson, K., Humphreys, R. M., Hillier, D. J., \& Ishibashi, K. 2006a, ApJ, 640, 474
Martin, J. C., Davidson, K., Humphreys, R. M., \& Mehner, A. 2010, AJ, 139 2056

Martin, J. C., Davidson, K., \& Koppelman, M. D. 2006b, AJ, 132, 2717

Martin, J. C., \& Koppelman, M. D. 2004, AJ, 127, 2352

Meaburn, J., Wolstencroft, R. D., \& Walsh, J. R. 1987, A\&A, 181, 333

Mehner, A. 2011, PhD thesis, Univ. Minnesota, USA

Mehner, A., Davidson, K., \& Ferland, G. J. 2011, ApJ, 737, 70

Mehner, A., Davidson, K., Ferland, G. J., \& Humphreys, R. M. 2010a, ApJ, 710,729

Mehner, A., Davidson, K., Humphreys, R. M., et al. 2010b, ApJ, 717, L22

Nielsen, K. E., Corcoran, M. F., Gull, T. R., et al. 2007, ApJ, 660, 669

Okazaki, A. T., Owocki, S. P., Russell, C. M. P., \& Corcoran, M. F. 2008, MNRAS, 388, L39

Osterbrock, D. E., \& Ferland, G. J. 2006, in Astrophysics of Gaseous Nebulae and Active Galactic Nuclei, ed. D. E. Osterbrock \& G. J. Ferland (Sausalito, CA: Univ. Science Books)

Owocki, S. 2005, in ASP Conf. Ser. 332, The Fate of the Most Massive Stars, ed. R. Humphreys \& K. Stanek (San Francisco, CA: ASP), 169

Parkin, E. R., Pittard, J. M., Corcoran, M. F., Hamaguchi, K., \& Stevens, I. R. 2009, MNRAS, 394, 1758

Pittard, J. M., \& Corcoran, M. F. 2002, A\&A, 383, 636

Richardson, N. D., Gies, D. R., Henry, T. J., Fernández-Lajús, E., \& Okazaki, A. T. 2010, AJ, 139, 1534

Sirianni, M., Jee, M. J., Benítez, N., et al. 2005, PASP, 117, 1049

Smith, N. 2006, ApJ, 644, 1151

Smith, N., Davidson, K., Gull, T. R., Ishibashi, K., \& Hillier, D. J. 2003, ApJ, 586,432

Soker, N. 2003, ApJ, 597, 513

Soker, N. 2007, ApJ, 661, 482

Soker, N., \& Behar, E. 2006, ApJ, 652, 1563

Stahl, O., Weis, K., Bomans, D. J., et al. 2005, A\&A, 435, 303

Steiner, J. E., \& Damineli, A. 2004, ApJ, 612, L133

Stevens, I. R., Blondin, J. M., \& Pollock, A. M. T. 1992, ApJ, 386, 265

Stevens, I. R., \& Kallman, T. R. 1990, ApJ, 365, 321

Stevens, I. R., \& Pittard, J. M. 1999, in ASP Conf. Ser. 179, Eta Carinae at The Millennium, ed. J. A. Morse, R. M. Humphreys, \& A. Damineli (San Francisco, CA: ASP), 295

Teodoro, M., Damineli, A., Arias, J. I., et al. 2011, arXiv:1104.2276v1

van Genderen, A. M., Sterken, C., Allen, W. H., \& Liller, W. 2003, A\&A, 412, L25

Viotti, R., Rossi, L., Cassatella, A., Altamore, A., \& Baratta, G. B. 1989, ApJS, 71,983

Weis, K., Stahl, O., Bomans, D. J., et al. 2005, AJ, 129, 1694

Whitelock, P. A., Feast, M. W., Koen, C., Roberts, G., \& Carter, B. S. 1994, MNRAS, 270, 364

Whitelock, P. A., Feast, M. W., Marang, F., \& Breedt, E. 2004, MNRAS, 352 , 447

Zanella, R., Wolf, B., \& Stahl, O. 1984, A\&A, 137, 79

Zethson, T. 2001, PhD thesis, Lunds Univ., Sweden

Zethson, T., Johansson, S., Davidson, K., et al. 1999, A\&A, 344, 211 